\title{
Very Massive Stars in the local Universe
}

Jorick S. Vink ${ }^{1}$, Alexander Heger ${ }^{2}$, Mark R. Krumholz ${ }^{3}$, Joachim Puls ${ }^{4}$, S. Banerjee ${ }^{5}$, N. Castro ${ }^{6}$, K.-J. Chen ${ }^{7}$, A.-N. Chenè ${ }^{8,9}$, P. A. Crowther ${ }^{10}$, A. Daminelli ${ }^{11}$, G. Gräfener ${ }^{1}$, J. H. Groh ${ }^{12}$, W.-R. Hamann ${ }^{13}$, S. Heap ${ }^{14}$, A. Herrero ${ }^{15}$, L. Kaper ${ }^{16}$, F. Najarro ${ }^{17}$, L. M. Oskinova ${ }^{13}$, A. Roman-Lopes ${ }^{18}$, A. Rosen ${ }^{3}$, A. Sander ${ }^{13}$, M. Shirazi ${ }^{19}$, Y. Sugawara ${ }^{20}$, F. Tramper ${ }^{16}$, D. Vanbeveren ${ }^{21}$, R. $\operatorname{Voss}^{22}$, A. Wofford
and the participants of JD2

${ }^{1}$ Armagh Observatory, College Hill, BT61 9DG, Armaghm Northern Ireland, UK email: jsv@arm.ac.uk

${ }^{2}$ Monash Centre for Astrophysics School of Mathematical Sciences, Building 28, M401 Monash University, Vic 3800, Australia

${ }^{3}$ Department of Astronomy \& Astrophysics, University of California, Santa Cruz, CA 95064, USA

${ }^{4}$ Universitäts-Sternwarte, Scheinerstrasse 1, 81679, Munchen, Germany

${ }^{5}$ Argelander-Institut für Astronomie, Auf dem Hügel 71, D-53121, Bonn, Germany

${ }^{6}$ Institute of Astronomy \& Astrophysics, National Observatory of Athens, I. Metaxa \& Vas. Pavlou St. P. Penteli, 15236 Athens, Greece

${ }^{7}$ Minnesota Institute for Astrophysics, University of Minnesota, Minneapolis, MN 55455, USA

${ }^{8}$ Departamento de Física y Astronomía, Universidad de Valparaíso, Av. Gran Bretaña 1111, Playa Ancha, Casilla 5030, Chile

${ }^{9}$ Departamento de Astronomía, Universidad de Concepción, Casilla 160-C, Chile

${ }^{10}$ Dept. of Physics \& Astronomy, Hounsfield Road, University of Sheffield, S3 7RH, UK

${ }^{11}$ Departamento de Astronomia do IAG-USP, R. do Matao 1226, 05508-090 Sao Paulo, Brazil

${ }^{12}$ Geneva Observatory, Geneva University, Chemin des Maillettes 51, CH-1290 Sauverny, Switzerland

${ }^{13}$ Institute for Physics and Astronomy, University Potsdam, 14476 Potsdam, Germany

${ }^{14}$ NASA Goddard Space Flight Center, Greenbelt, MD 20771, USA

${ }^{15}$ Instituto de Astrofisica de Canarias and Universidad de La Laguna, E-38200 La Laguna, Spain

${ }^{16}$ Astronomical Institute 'Anton Pannekoek', University of Amsterdam, Science Park 904, PO Box 94249, 1090 GE Amsterdam, The Netherlands

${ }^{17}$ Departamento de Astrofsica, Centro de Astrobiologia, (CSIC-INTA), Ctra. Torrejn a Ajalvir, $\mathrm{km}$ 4, 28850 Torrejon de Ardoz, Madrid, Spain

${ }^{18}$ Physics Department - Universidad de La Serena - Av. Cisternas, 1200 - La Serena - Chile

${ }^{19}$ Leiden Observatory, Leiden University, P.O. Box 9513, 2300 RA Leiden, The Netherlands

${ }^{20}$ Department of Physics, Faculty of Science \& Engineering, Chuo University, 1-13-27 Kasuga, Bunkyo, Tokyo 112-8551

${ }^{21}$ Astrophysical Institute, Vrije Universiteit Brussel, Pleinlaan 2, 1050, Brussels, Belgium

${ }^{22}$ Department of Astrophysics/IMAPP, Radboud University Nijmegen, PO Box 9010, NL-6500 GL Nijmegen, the Netherlands

${ }^{23}$ Space Telescope Science Institute, 3700 San Martin Drive, Baltimore, MD, 21218, USA

${ }^{24}$ Department of Astronomy, University of Florida, Gainesville, FL 32611, USA

\begin{abstract}
Recent studies have claimed the existence of very massive stars (VMS) up to $300 M_{\odot}$ in the local Universe. As this finding may represent a paradigm shift for the canonical stellar upper-mass limit of $150 M_{\odot}$, it is timely to discuss the status of the data, as well as the farreaching implications of such objects. We held a Joint Discussion at the General Assembly in Beijing to discuss (i) the determination of the current masses of the most massive stars, (ii) the formation of VMS, (iii) their mass loss, and (iv) their evolution and final fate. The prime aim
\end{abstract}


was to reach broad consensus between observers and theorists on how to identify and quantify the dominant physical processes.

Keywords. Stars: massive stars, Stars: mass-loss, Stars: stellar evolution

\section{Introduction}

The last decade has seen a growing interest in the study of the most massive stars, as their formation seems to be favourable in the early Universe at low metalliciy $(Z)$, and is thought to involve a population of objects in the range 100-300 $M_{\odot}$ (Bromm et al. 1999; Abel et al. 2002). The first couple of stellar generations may be good candidates for the reionization of the Universe. Notwithstanding the role of the first stars, the interest in the current generation of massive stars has grown as well. Massive stars are important drivers for the evolution of galaxies, as the prime contributors to the chemical and energy input into the interstellar medium (ISM) through stellar winds and supernovae (SNe). A number of exciting developments have recently taken place, such as the detection of a long-duration gamma-ray burst (GRB) at a redshift of 9.4, just a few hundred millions years after the Big Bang (Cucchiara et al. 2011). This provides convincing evidence that massive stars are able to form and die massive when the Universe was not yet enriched.

The specific reason for holding this JD was the recent evidence for the existence and subsequent deaths of very massive stars (VMS) up to $300 M_{\odot}$. Gal-Yam et al. (2009) claimed the detection of a pair-instability SN (PSN) from a VMS. These explosions are thought to disrupt stars without leaving any remnants. Crowther et al. (2010) reanalyzed the most massive hydrogen-and nitrogen-rich Wolf-Rayet (WNh) stars in the center of R136, the ionizing cluster of the Tarantula nebula in the Large Magellanic Cloud (LMC). The conclusion from their analysis was that stars usually assumed to be below the canonical stellar upper-mass limit of $150 M_{\odot}$ (of e.g. Figer 2005), were actually found to be much more luminous, and with initial masses up to $\sim 320 M_{\odot}$. Prior to discussing the formation, evolution, and fate of VMS, and before we should explore the full implications of these findings, it is imperative to discuss the various lines of evidence for and against VMS.

VMS are usually found in and around young massive clusters, such as the Arches cluster in the Galactic centre and the local starburst region R136 in the LMC. Such clusters may harbor intermediate-mass black holes (IMBHs) with masses in the range of several 100 to several $1000 M_{\odot}$ and may provide insight into the formation of supermassive black holes of order $10^{5} M_{\odot}$. Young clusters are also relevant for the unsolved problem of massive star formation.

For decades it was a struggle to form stars over $10 M_{\odot}$, as radiation pressure on dust grains might halt and reverse the accretion flow onto the central object (e.g., Yorke \& Kruegel 1977). Because of this problem, astrophysicists have been creative in forming massive stars via competitive accretion and merging in dense cluster environments (e.g., Bonnell et al. 1998). In more recent times several multi-D simulations have shown that massive stars might form via disk accretion after all (e.g., Krumholz et al. 2009; Kuiper et al. 2010). In the light of recent claims for the existence of VMS in dense clusters, however, we should redress the issue of forming VMS in extreme environments.

Massive clusters may be so dense that their early evolution is largely affected by stellar dynamics, and possibly form very massive objects via runaway collisions (e.g., Portegies Zwart et al. 1999; Gürkan et al. 2004), leading to the formation of VMS up to $1000 M_{\odot}$ at the cluster center, which may produce IMBHs at the end of their lives, but only if 
VMS mass loss is not too severe (see Belkus et al. 2007, Yungelson et al. 2008, Glebbeek et al. 2009, Pauldrach et al. 2012).

VMS are thought to evolve almost chemically homogeneously (Gräfener et al. 2011), implying that knowing the exact details of the mixing processes (e.g., rotation, magnetic fields) could be less relevant in comparison to their canonical $\sim 10-60 M_{\odot}$ counterparts. Instead, the evolution and death of VMS is likely dominated by mass loss. A crucial issue regards the relevance of episodes of super-Eddington, continuum-driven mass loss (such as occurs in Eta Carinae and other Luminous Blue Variable (LBV) star eruptions), which might be able to remove large amounts of mass - even in the absence of substantial line-driven winds (see Sect.6).

A final issue concerns the fate of VMS, and more specifically whether VMS end their lives as canonical Wolf-Rayet (WR) stars giving rise to Type Ibc SNe, or do they explode prematurely during the LBV phase? Might some of the most massive stars even produce PSNe? And how do PSNe compare to the general population of super-luminous SNe (SLSNe) that have recently been unveiled by Quimby et al. (2011), and are now seen out to high redshifts (Cooke et al. 2012). Such spectacular events can only be understood once we have obtained a basic knowledge of the physics of VMS.

In Section 2 a definition for VMS is adopted, before we discuss the evidence for and against super-canonical stars, i.e. objects above the traditional $150 M_{\odot}$ stellar upper mass limit (Sect. 3). After it is concluded that VMS probably exist, the next question to address is how to form such objects (Sect.4). We then discuss the properties of VMS in Sect. 5, before discussing their mass loss (Sect.6), evolution and fate (Sect.7). We end with the implications (Sect. 8) and final words.

\section{Definition of VMS}

Before we can discuss the evidences for and against VMS, one of the very first issues we discussed during the JD was what actually constitutes a "very" massive star. One may approach this in several different ways. Theoretically, "normal" massive stars with masses above $\sim 8 M_{\odot}$ are those that produce core-collapse SNe (Smartt et al. 2009), but what happens at the upper-mass end (Nomoto 2012)?

Above a certain critical mass, one would expect the occurrence of PSNe, and ideally this could be the lower-mass limit for the definition of our VMS. However, in practice this number is not known a priori due to mass loss, and as a result the initial and final masses are not the same. In other words, the initial main-sequence mass for PSN formation is model-dependent, and thus somewhat arbitrary. Moreover, there is the complicating issue of pulsational pair-instability supernova (Puls-PSN) at masses below those of full-fedged PSNe (e.g. Woosley et al. 2007). One could of course resort to the mass of the helium (He) core for which objects reach the conditions of electron/positron pair-formation instability. Heger showed this minimum mass to be $\sim 40 M_{\odot}$ to encounter Puls-PSN and $\sim 65 M_{\odot}$ to encounter PSNe (see also Chatzopoulus \& Wheeler 2012).

An alternative definition could involve the spectroscopic transition between normal main-sequence O-type stars and hydrogen-rich Wolf-Rayet stars (of WNh type), which have also been shown to be core $\mathrm{H}$ burning main-sequence objects. However, such a definition would also be model dependent.

For these reasons, we took the decision to follow a more pragmatic approach: we consider stars to be very massive when the initial masses are $\sim 100 M_{\odot}$, or higher. 


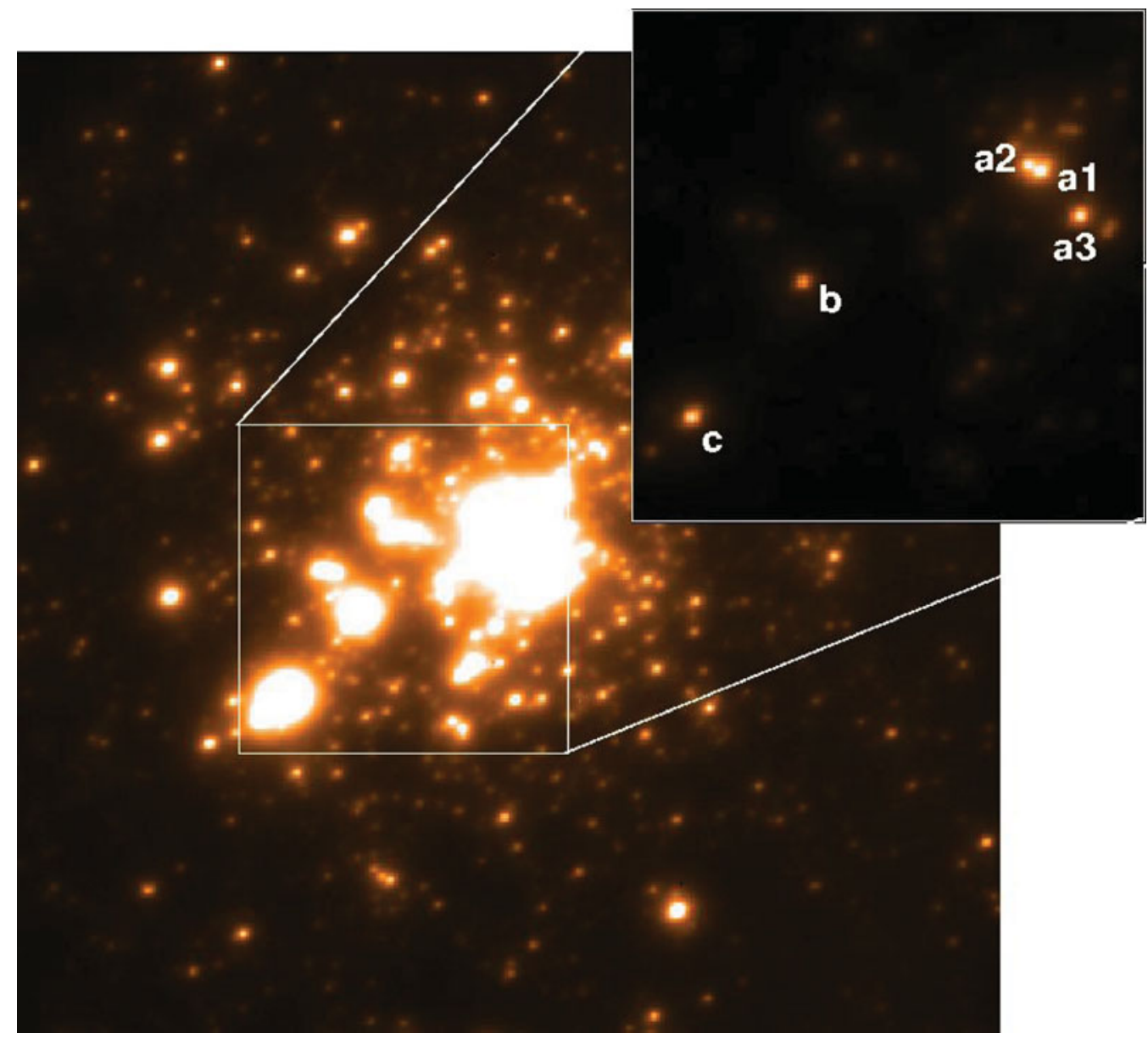

Figure 1. VLT MAD $\mathrm{K}_{s}$-band $12 \times 12 \operatorname{arcsec}(3 \times 3$ parsec for the LMC distance of $49 \mathrm{kpc})$ image of R136 (Campbell et al. (2010) in conjunction with a view of the central $4 \times 4$ arcsec $(1 \times 1$ parsec) in which the very massive WN5h stars discussed are labelled (component b is a lower mass WN9h star). Relative photometry agrees closely with integral field SINFONI observations of Schnurr et al. 2009). See Crowther et al. (2010) for details.

\section{On the existence of VMS}

With this definition, the question of whether very massive stars exist can convincingly be addressed with the one answer: yes they do! However, the pertinent issue for our JD was whether the widely held "canonical" upper-mass limit of $150 M_{\odot}$ has recently been superseded.

Paul Crowther gave the first invited review presenting evidence for initial masses as high as $320 M_{\odot}$. Crowther provided historical context to some of the astronomical community's sceptism regarding such high masses in R136, in particular the spectacular claim for the existence of a $2500 M_{\odot}$ star R136 in the 30 Doradus region of the LMC some 3 decades ago (e.g. Cassinelli et al. 1981). Higher spatial resolution showed that R136 was actually not a single supermassive star, but the object broke up into a young cluster containing significantly lower mass objects, including the current record holder R136a1 (as well as R136a2, R136a3, and R136c). Therefore, over the last few decades, there was a consensus of a $150 M_{\odot}$ stellar upper mass limit (Weidner \& Kroupa 2004; Figer 2005; 
Oey \& Clarke 2005, Koen 2006), although the accuracy of this magic number of 150 was low (Massey 2011).

Crowther et al. (2010) re-analyzed the photometric and spectroscopic data of the VMS in R136. In comparison to the older WFPC2 data, they used ground-based adaptive optics photometry (see Fig. 1). In combination with their spectral analysis using the CMFGEN non-LTE atmosphere code of Hillier \& Miller (1998), this lead to higher estimates for effective temperatures and bolometric corrections. In conclusion they claimed that the R136 cluster hosts several stars with masses as high as 200-300 $M_{\odot}$.

Crowther et al. also performed a "sanity check" on similar WNh objects in the Galactic starburst cluster NGC 3601. Although these objects were fainter, and less massive, than those in R136, the advantage was the available dynamical mass estimate by Schnurr et al. (2008) of the binary object NGC 3601-A1 of $116 \pm 31+89 \pm 16 M_{\odot}$. This is important as the least model-dependent way to obtain stellar masses is through the analysis of the light-curves and radial velocities induced by binary motions. We also note that Rauw et al. (2004) and Bonanos et al. (2004) found both components of the eclipsing WolfRayet binary WR20a to be particularly massive, with $83 \pm 5$ and $82 \pm 5 M_{\odot}$, with small error bars.

During the lively discussion that followed Paul's review, some attendants argued that the luminosities derived by Crowther et al. are uncertain and these "single" objects might actually contain multiple sources. Whilst short-period binaries were not detected by Schnurr et al. (2009), longer period binaries are harder to exclude. One of the additional arguments by Crowther et al. was that X-rays have not been detected, whilst they may have been expected on the basis of colliding wind binary (CWB) simulations by Pittard \& Stevens (2002). Oskinova however countered this argument on the basis that empirically a low X-ray luminosity cannot serve as a robust argument against a binary nature (see Sect. 6).

Najarro noted that even the best image so far of the Arches cluster with Keck (Fig. 2). has a limited spatial resolution of 50 milli-arcsec (mas), which corresponds to roughly $1 / 10$ of the diameter of the circles marking the PSF reference stars in the figure. Given that the LMC is almost 7 times further away, and that the VLT is smaller than Keck, the circles from Fig. 2 would roughly correspond to the spatial resolution achieved with the VLT if the Arches clusters was in the LMC. In other words, the Arches stars would effectively "merge" with surrounding objects. This analogy suggests that we cannot exclude the possibility that the bright WNh stars in the R136 cluster core could still break up into several lower-mass WNh stars. However, for example a $\sim 300 M_{\odot}$ star could at best break up into a pair of $\sim 150 M_{\odot}$ stars (given the shallow slope of the stellar luminosity to mass ratio at the high-mass end).

Moreover, Bestenlehner noted that there is a near-identical twin of R136a3 WNh star in 30 Doradus: VFTS 682. Its key relevance is that it is found in isolation from the R136 cluster, some 30 pc away. For this object line-of-sight contamination is far less likely than for the R136 core stars. VFTS 682 thus offers another sanity check on the reliability of the luminosities for the R136 core stars. Bestenlehner et al. (2011) derived a high luminosity of $\log \left(L / L_{\odot}\right)=6.5$ and a present-day mass of $150 M_{\odot}$ for VFTS 682. This implied an initial mass on the zero-age main sequence (ZAMS) higher than the canonical upper-mass limit - likely $\sim 200 M_{\odot}$.

In his talk Hamann showed results from another extremely luminous WN star in the Galactic center region: the Peony star (Barniske et al. 2008). The luminostiy of this star is determined from spectral analysis as $\log \left(L / L_{\odot}\right)=6.5 \pm 0.2$ and initial mass between $150 \leqslant M_{\mathrm{u}} / M_{\odot} \leqslant 200$. The star is located above the Humphreys-Davidson limit, in the region populated by the LBV stars. However, the hydrogen content is lower in WR 102ka 


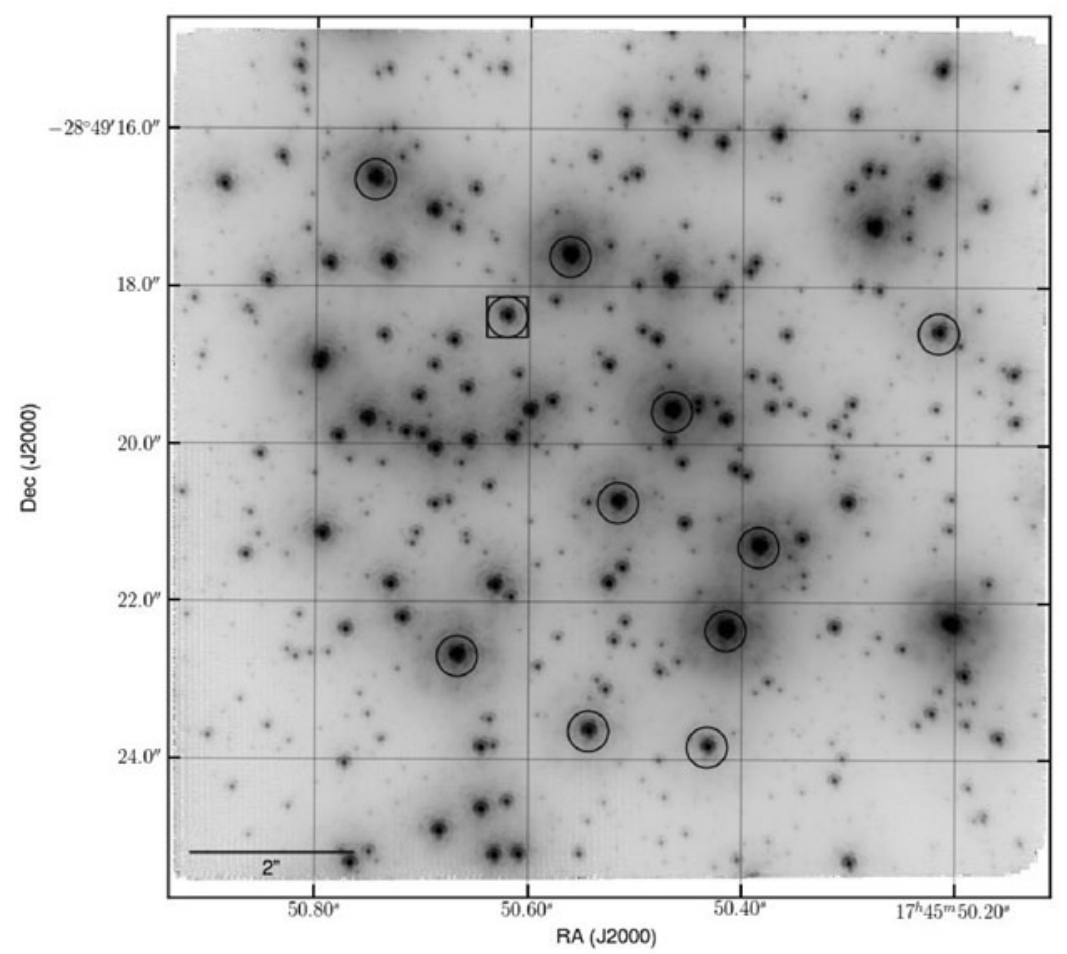

Figure 2. K-band mosaic of the core field of the Galactic Arches cluster at a resolution of 50 mas and with a sensitivity of $K_{\lim }=20.6 \mathrm{mag}$. The figure is taken from Clarkson et al. (2012).

compared to the Pistol star, while helium is higher. This indicates a more advanced evolutionary stage of the former, compared to "normal" LBV stars.

In summary, whilst one cannot exclude that the object R136a1 claimed to be $\sim 300 M_{\odot}$ in the R136 cluster might still turn out to "dissolve" when higher spatial resolution observations were to become available, a number of sanity checks involving binary dynamics and "isolated" objects make it rather convincing that stars with ZAMS masses of $200 M_{\odot}$ exist. In any case, no fundamental reason was identified why "150" would be a magic number.

\section{Formation of VMS}

The key question to address was what is so special about the formation of $250 M_{\odot}$ stars in comparison to 'normal' $50 M_{\odot}$ O-type stars? Can these VMS only form inside dense cluster environments? Other relevant questions involve the time-scale and evolutionary stages when they finish formation/accretion. How fast do they rotate? What VMS fraction is in binaries, and what are the binary properties? Are the most massive stars all the result of stellar mergers?

\subsection{Theoretical considerations}

According to our second invited review speaker, Mark Krumholz, VMS formation is not a fundamentally different problem from the formation of massive stars in general. From an observational standpoint VMS in clusters appear as part of a continuous initial mass function (IMF), with no special features that mark them off as different from the remainder of the stellar population. From a theoretical standpoint, both the radiative 
and wind luminosities of stars are increasing functions of mass, but from the standpoint of star formation there is no natural dividing line that would put a star of, for example, $50 M_{\odot}$ into one category and a star of $250 M_{\odot}$ into another. For this reason, it makes sense not to treat the formation of very massive stars as a separate problem, and instead to embed it in the broader context of forming the upper end of the IMF.

There are three main challenges to forming massive stars: fragmentation, binarity, and radiation pressure. The fragmentation problem is simple to state: we certainly see gas over-densities ("cores") with masses of $\sim 100 M_{\odot}$ or larger and radii of $\sim 0.1 \mathrm{pc}$ or less that seem promising sites for massive star formation (e.g. Beuther et al. 2005; Bontemps et al. 2010). However, given these masses and radii, and typical molecular cloud temperatures of $\sim 10 \mathrm{~K}$, the Jeans mass is below $1 M_{\odot}$, so how can the objects avoid fragmenting and collapse to form an object containing 100 Jeans masses or more? The answer seems to be that the classical Jeans analysis of an isothermal gas does a rather poor job at predicting the behavior of a radiatively heated, magnetized fluid. Once small stars form and begin to accrete, their radiation heats the gas around them and suppresses fragmentation in it (Krumholz 2006; Krumholz et al. 2007; Krumholz \& McKee 2008). Magnetic fields also make it much more difficult for the gas to fragment (Hennebelle et al. 2011), and the combined effects of radiation and magnetic fields seems to be particularly effective, as seen in Fig. 3 (Commerçon et al. 2011; Myers et al. 2012). These effects together seem to resolve the fragmentation problem, indicating that massive stars can form from the direct collapse of massive protostellar cores with properties similar to those observed.

The second challenge is explaining why so many massive stars appear to be binaries (e.g. Sana et al. 2012). The observed multiplicity fraction rises very sharply with stellar mass, reaching near unity for O and B stars (excluding runaways - e.g. Brown 2001). Radiation-hydrodynamic simulations of star cluster formation appear able to replicate the observed dependence of multiplicity on primary mass (Bate 2012; Krumholz et al. 2012), and digging into the physical origin for this result indicates that it combines two effects. The first is simple N-body processing: close encounters between stars in young clusters tend to put the most massive members into binaries even if they are not born that way, while stripping companions from less massive stars. The second is disk fragmentation, as explored by (Kratter \& Matzner 2006) and (Kratter et al. 2008, 2010). Massive stars form with high accretion rates, and these accretion rates tend to produce disks with masses that can approach that of the primary. When this happens, disks are likely to fragment, and a common outcome of this process is that the disk produces a massive companion to the primary.

The third challenge is radiation pressure. Dusty interstellar gas has a high opacity, and the radiation force is proportional to this opacity. As a result, the radiative force exerted by a star's light can exceed its gravitational force for all stars larger than $\sim 20 M_{\odot}$ (Wolfire \& Cassinelli 1987; Krumholz et al. 2009). However, recent numerical and analytic work has shown that this problem is mostly an illusion; the radiation pressure barrier can be circumvented in numerous ways. First, in the presence of an accretion disk, radiation can be beamed away from the bulk of the accreting matter (Nakano et al. 1995; Jijina \& Adams 1996; Yorke \& Sonnhalter 2002; Kuiper et al. 2010, 2011). Second, radiationdriven Rayleigh-Taylor instabilities can break up bubbles of radiation and allow matter to accrete through optically-thick fingers (Krumholz et al. 2009; Jacquet \& Krumholz 2011). Figure 4 shows an example. Third, protostellar jets can punch holes in accreting cores that allow radiation to leak out, reducing the net radiation force in most directions and allowing accretion to continue even though, averaged over $4 \pi$ sr, the radiation force is stronger than gravity (Krumholz et al. 2005; Cunningham et al. 2011). The takeaway 


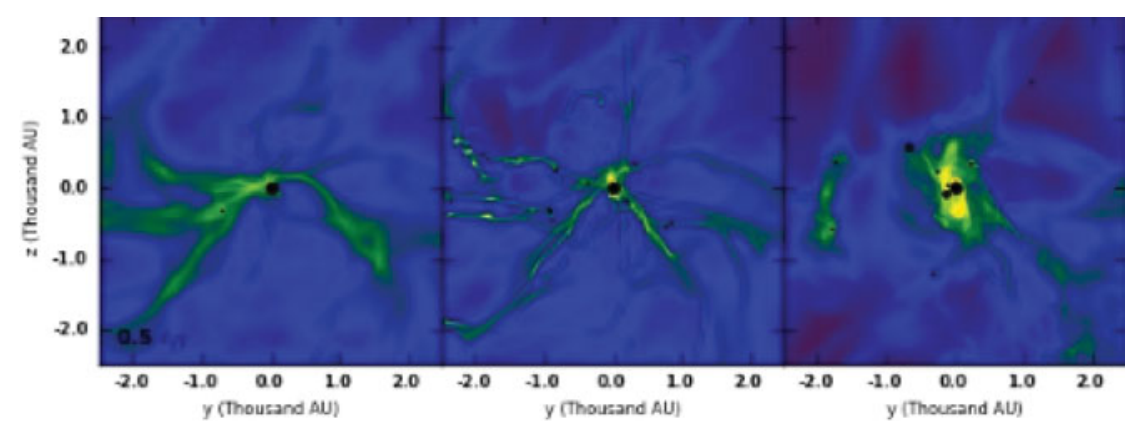

Figure 3. Column density projections from three simulations of a collapsing high mass core, from (Myers et al. 2012). All three simulations use identical initial conditions, but the leftmost one includes radiation and magnetic fields, the center one includes magnetic fields but not radiative transfer, and the rightmost one includes radiation but not magnetic fields. Note the dramatic reduction in number of stars (black circles) in the radiation plus magnetic fields case. The color scale runs from $10^{-0.75}-10^{3.25} \mathrm{~g} \mathrm{~cm}^{-2}$.

message from all of these simulations is that radiation pressure poses no barrier to the formation of stars up to arbitrary masses.

Given that modern theoretical models have removed all of the serious objections to forming massive stars in the same way that low mass stars form, i.e. by accretion from a collapsing gas core, there is no need to resort to exotic processes like stellar collisions (e.g. Bonnell et al. 1998). This does not mean, however, that stellar collisions cannot happen and also contribute to the massive star population. The question is: under what circumstances do we expect collisions to be important? The most recent and comprehensive papers to address this question are (Moeckel \& Clarke 2011) and (Baumgardt \& Klessen 2011) who both conducted N-body simulations of stars confined by a gaseous potential, and who came to similar conclusions. They find that the collisional formation of very massive stars is only significant if the stellar density is extremely high, in excess of $10^{7} \mathrm{pc}^{-3}$, with surface densities reaching $10^{5} \mathrm{pc}^{-2}$. These numbers are so high that even the Arches cluster would not be expected to have significant contributions to its massive star population by collisions. Moreover, if collisions are significant, they do not produce an IMF that looks like what we observe. Instead, because collisions tend to occur among the most massive stars, they produce an IMF that has a Salpeter-like slope at intermediate masses, then a deficit of stars at higher masses, and finally one or a few very massive stars. IMFs with dips of this sort have not been observed, again suggesting that collisions are not likely to be significant contributors to the massive star populations.

\subsection{The potential role of dynamically induced mergers}

An alternative VMS formation scenario was presented by Sambaran Banerjee (with Pavel Kroupa and Seungkyung Oh; Banerjee et al. 2012a) who argued that super-canonical stars can be formed out of a dense stellar population - with a canonical IMF and with a $150 M_{\odot}$ upper limit - through dynamically induced mergers of the most massive binaries. Banerjee et al. performed direct N-body computations (NBODY6; Aarseth 2003) of a fully mass-segregated star cluster mimicking R136 in which all the massive stars are in primordial binaries. Banerjee et al. account for the mass evolution of the super-canonical stars and the resulting shortened $(\approx 1.5 \mathrm{Myr})$ lifetimes in their super-canonical phases using stellar evolutionary models by Köhler \& Langer (2012) that incorporate Vink et al. (2000) mass-loss rates for the main sequence and Hamann et al. (1995) for the He burning WR phase. 

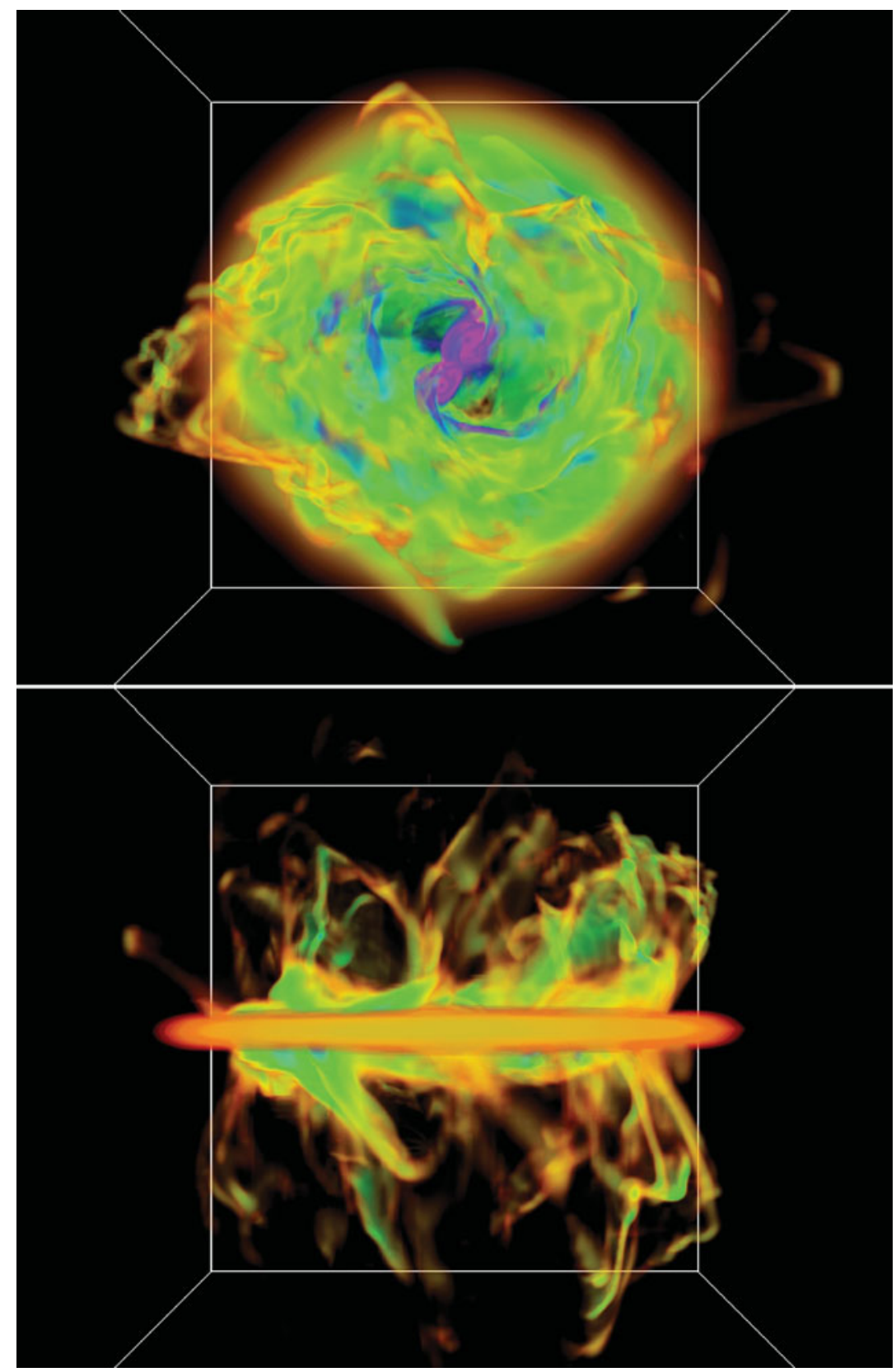

Figure 4. Volume renderings of a simulation from (Krumholz et al. 2009) involving the density field in a $(4000 \mathrm{AU})^{3}$ region at $55.0 \mathrm{kyr}$ of evolution. The color scale is logarithmic and runs from $10^{-16.5}-10^{-14} \mathrm{~g} \mathrm{~cm}^{-3}$. The left panel shows the polar view, and the right one denotes the edge-on view. The figure highlights how Rayleigh-Taylor instability fingers channel matter onto a massive binary star system.

Banerjee et al. find that super-canonical stars begin to form via dynamical mergers of massive binaries from $\approx 1$ Myr cluster age, obtaining stars with initial masses up to $\approx 250 M_{\odot}$. Multiple super-canonical stars are found to remain bound to the cluster simultaneously within a super-canonical lifetime. Banerjee also noted that some of these 
objects can be formed at runaway velocities which escape the cluster at birth. For instance, the most massive apparently isolated WNh star VFTS 682 might be an expected slow runaway (Banerjee et al. 2012b; see also Fujii \& Portegies-Zwart 2012).

The Banarjee et al. models indicate that had super-canonical stars formed primordially alongside the rest of the R136 cluster, i.e. violating the canonical upper limit, they would have evolved below the canonical $150 M_{\odot}$ limit by $\approx 3 \mathrm{Myr}$, the likely age of the bulk of R136 according to Andersen et al. (2009). In other words, Banerjee et al. argue that primordially-formed super-canonical stars should not be observed at the present time in R136, whilst it is quite plausible that a collection of dynamically formed super-canonical VMS would be observed in the centres of young massive starburst clusters.

A fully self-consistent N-body computation incorporating detailed accurate evolutionary and mass-loss recipes would be needed to confirm these scenarios.

\subsection{Rotation rates as a constraint on massive star formation}

Returning to the more conventional ways of forming massive stars, Anna Rosen addressed the question of what sets the initial rotation rates of massive stars. The physical mechanisms that set the initial massive star rotation rates are a crucial unknown in current star-formation theory. Observations of young, massive stars provide evidence that they form in a similar fashion to their lower mass counterparts. The magnetic coupling between a star and its accretion disk may be sufficient to spin down low-mass pre-main-sequence (PMS) stars to well below breakup at the end stage of their formation when the accretion rate is low. However, Anna showed that these magnetic torques are insufficient to spin down massive PMS stars due to their short formation times and high accretion rates. Anna developed a model for the angular momentum evolution of stars over a wide range in mass, considering both magnetic and gravitational torques. She finds that magnetic torques are unable to spin down either low-mass or high-mass stars during the main accretion phase, and that massive stars cannot be spun down significantly by magnetic torques during the end stage of their formation either. Spin-down occurs only if massive stars' disk lifetimes are substantially longer or their magnetic fields are much stronger than current observations suggest (Rosen et al. 2012).

\subsection{Observations of massive star formation}

Heavy extinction hides the birthplaces of massive stars from view, and the short formation timescales set a strong limitation to the sample of objects that can be studied. So far, our physical knowledge of massive young stellar objects (YSOs) has been derived from near-IR imaging and spectroscopy, revealing populations of young OB-type stars, some still surrounded by a disk, others apparently 'normal' main sequence stars powering H II regions. The most important spectral features of OB-type stars are however located in the ultraviolet (UV) and optical range.

Kaper (with Ellerbroek, Ochsendorf and Bik) showed that with the new optical/nearinfrared spectrograph X-shooter on ESO's Very Large Telescope (VLT), it is possible to extend the spectral coverage of these massive YSOs into the optical range. First results are very promising, although they seem to probe the intermediate-mass $\left(\sim 2-8 \mathrm{M}_{\odot}\right)$ range rather than the massive star range. Ellerbroek et al. (2011) discovered a jet (HH 1042) produced by the massive YSO nr292 in the massive star forming region RCW 36, demonstrating that the object is still actively accreting. The mass of this star is likely less than $6 \mathrm{M}_{\odot}$ and remains uncertain as photospheric features are not detected. The first firm spectral classification of B275, a massive YSO in M17, results in its precise location on a PMS track for a $\sim 7 M_{\odot}$ star (see Fig. 5; Ochsendorf et al. 2011), has the size of a 


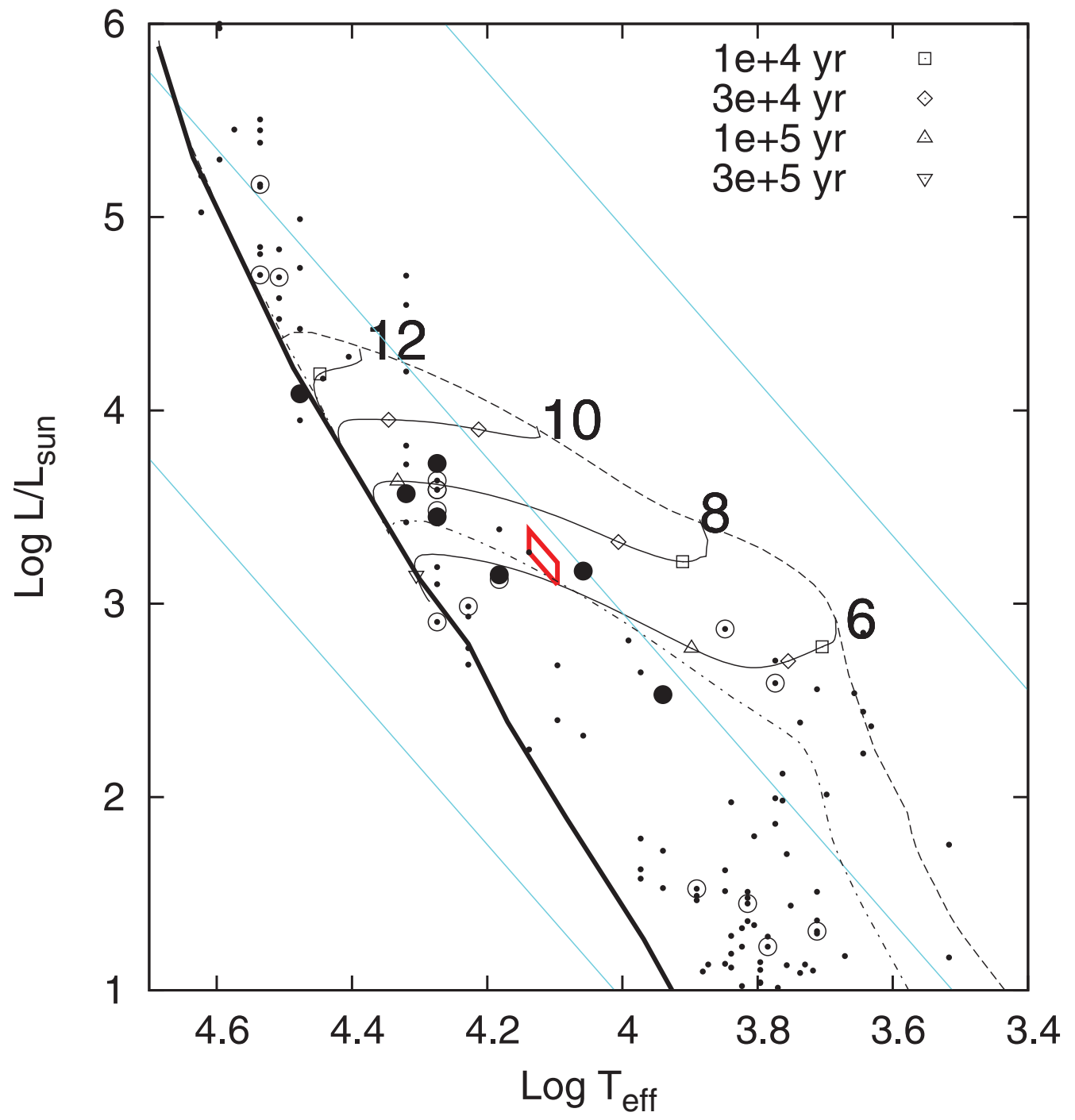

Figure 5. The location of B275 (red parallelogram) in the HRD next to PMS tracks from Hosokawa et al. (2010) with the ZAMS mass labeled and open symbols indicating lifetimes. The thin dashed and thin dot-dashed lines are the birth lines for accretion rates of $10^{-4} \mathrm{M}_{\odot} \mathrm{yr}^{-1}$ and $10^{-5} \mathrm{M}_{\odot} \mathrm{yr}^{-1}$, respectively; the thick solid line is the ZAMS (Schaller et al. 1992). The filled and open circles represent stars in M 17 for which a spectral type has been determined (Hoffmeister et al. 2008); dots are other stars in M 17. B275 is on its way to becoming a $68 M_{\odot}$ ZAMS star, so far one of the most massive pre-main-sequence star known. The figure has been adapted from Ochsendorf et al. (2011).

bloated giant, as predicted by models of Hosokawa et al. (2010), and is still surrounded by a disk.

It remains unclear whether the progenitors of the most massive VMS will be detectable this way.

\subsubsection{Radiation Transfer Modeling: From massive to very massive star formation}

In order to properly interpret the observations of massive protostars, Zhang et al. (with Tan, McKee, and de Buizer) presented a radiation transfer (RT) model for a massive core 
in a high pressure environment that forms a massive star through core accretion (Zhang \& Tan 2011; Zhang, Tan \& McKee, 2012, ApJ submitted). This RT model is based on the Turbulent Core model of McKee \& Tan (2003): massive stars form in dense clumps with high surface density. Assuming the rotational-to-gravitational energy ratio is $2 \%$ in the core, whilst the disk has a diameter of $\sim 1000 \mathrm{AU}$. The high accretion rate leads to a disk mass comparable to the stellar mass. In Zhang et al. (2012), the treatment of the disk was improved by allowing radially varying accretion rates due to a supply of mass and angular momentum from the infall envelope and their loss to the disk wind. The transfer of accretion power to mechanical power of the wind was also accounted for. An approximate disk wind solution was developed partly based on the Blandford \& Payne (1982) model. The simulation was performed with the latest version of the Monte Carlo RT code by Whitney et al. (2003). Corrections made by adiabatic cooling/heating and advection were included, and so were the gas opacities.

The model was compared to the massive protostar G35.2-0.74N. At a distance of $2.2 \mathrm{kpc}$, radio continuum emission indicates there is a bipolar outflow from this source. In recent SOFIA-FORCAST observations of the massive protostar G35.2-0.74N at 31 and $37 \mathrm{~m}$ (Zhang et al. 2012, in prep.) both the near- and far-facing sides of the outflow can be seen. The latter is missing in mid-IR continuum. By fitting both the observed SED and the outflow-axis intensity profiles with the RT model, the bolometric luminosity was inferred to be $\sim 10^{5} L_{\odot}$ after correcting for foreground extinction and the dependence of luminosity on inclination (flashlight effect).

The fitting model suggests a massive $\sim 30 M_{\odot}$ protostar is forming from a $\sim 240 M_{\odot}$ core with a high surface density of $\sim 1 \mathrm{~g} / \mathrm{cm}^{2}$, via relatively ordered collapse and accretion, and driving powerful bipolar outflows. These results seem to support the core accretion theory which predicts massive stars may form in similar ways to their low-mass counterparts. Simply extending the model to protostars with higher masses up to $100 M_{\odot}$, Zhang et al. noticed a shift of the SED peaks to shorter wavelengths and a change of mid-IR slope. The flashlight effect turns out to be huge for such sources. In the mid-IR, the luminosity from face-on view can be higher than that from edge-on view by as much as 3 dex - suggesting that a large correction factor needs to be applied when using IR observations for deducing source luminosities.

\section{Properties of VMS}

Once a star has formed it starts to burn hydrogen on the ZAMS. For massive stars, the main sequence band probably consists of two spectroscopically distinct groups of objects: the $\mathrm{O}$ stars, and the WNh stars. The first group are thought to form the normal massive star sequence with masses of up to at least $\sim 60 M_{\odot}$. For the most massive "O stars on steroids" WNh (H-rich WR stars), their masses may be up to $300 M_{\odot}$. or higher.

The transition mass between $\mathrm{O}$ and $\mathrm{WNh}$ is not exactly known but is probably lies somewhere in the range $60-120 M_{\odot}$. Of course these numbers are model (mass loss and $Z$ ) dependent. The WNh stars are presumably in close proximity to the Eddington limit, and so are their descendant LBV and classical WN, WC, WO Wolf-Rayet stars. Some of these objects are thought to be in an evolutionary phase just prior to their final demise.

At the end of this section, we also consider extra-galactic VMS properties, both at very low (e.g. IC 1613) and high metal content (e.g. M33).

\subsection{WNh H-rich Wolf-Rayet stars - "O stars on steroids"}

WNh stars were discussed by Crowther and Hamann. In their analysis they make use of non-LTE model atmospheres, such as CMFGEN (Hillier \& Miller 1998) and PoWR 
(Hamann et al. 2006), whilst FASTWIND (Puls et al. 2005) is often used for normal O stars with weaker winds. These are 1D spherical symmetric codes that include lineblanketing and stellar winds allowing for micro-clumping (optically thick macro-clumping allowing for porosity is non-standard). Another important aspect of the analysis is that of accurate infrared photometry (see the infrared $\mathrm{SpS}$ on atmosphere modelling). This is especially true for those objects in the cores of dense clusters such as R136. The upshot of recent analyses is that WNh stars are more luminous, and more massive, than previously thought (see Sect.3). Hamann presented some early results from their spectral analysis of WN stars in the Magellanic Clouds (Hainich et al. in prep.) - complementing the published Galactic study of Hamann et al. (2006). Whilst the LMC objects are found to be bright with $\log L / L_{\odot}$ values in the range 5.3-5.9, Hamann warns that many of the brighter allegedly single WNh stars may actually be binaries, and not accounting for this fact may overestimate the luminosities of WNh stars.

\subsection{LBVs: unstable massive stars close to the Eddington limit}

Very massive stars are thought to evolve through the unstable Luminous Blue Variable phase, when enormous amounts of mass are lost. While LBVs have been classically thought to be rapidly evolving massive stars in the transitory phase from O-type to WolfRayet stars, recent studies have suggested that LBVs might surprisingly explode prematurely as a core-collapse supernova (Kotak \& Vink 2006, Gal-Yam et al. 2007, Mauerhan et al. 2012). Such a striking result highlights that the evolution of VMS through the LBV phase is far from being understood.

Groh discussed the recent advances in understanding LBVs, in particular how to distinguish them from the normal B supergiants and hypergiants (as discussed by Negueruela). LBVs can be recognized either from Giant Eruptions like Eta Car and P Cygni, or through their S-Dor variability, sometimes imprinted on peculiar looking double-peaked absorption profiles, in just a single epoch spectrum (Groh \& Vink 2011). LBVs do not always present both S Dor and giant outburst phenomena, leaving room for quite a heterogeneous class of objects.

Groh emphasized that LBVs are characterized on a phenomenological basis and, therefore, LBVs are neither a spectroscopic nor an evolutionary classification. Particular emphasis was given to describe the main properties of the S-Dor type variability (also performed by Stringfellow). These are changes in the hydrostatic radius and bolometric luminosity. Finally, the role of rapid rotation on LBVs was discussed (Groh et al. 2009).

\subsection{The Galactic WC stars}

WC star spectra are well known for their broad emission lines from helium, carbon and oxygen. Due to the absence of hydrogen these stars have to be core-helium burning and have as such been identified as the late evolutionary stage of massive and very massive stars. Sander et al. (2012) analyzed the optical and UV spectra of over 50 Galactic WC single stars and derived their parameters including their mass-loss rates. Sander et al. showed that the positions of the Galactic WC stars in the HR-diagram do not fit with the assumption that the most massive stars will pass the WC stage. Instead Sander et al. argue that their results indicate that WC stars come from an initial mass range between 20 and somewhere around $50 M_{\odot}$. The stellar evolution models of Vanbeveren et al. (1998) that include enhanced mass loss during the red sugergiant (RSG) phase appear to properly account for the location of the WC stars in the observed HR-diagram. It also seems that stars with higher initial masses do not reach the WC stage but instead explode after passing a WNL and probably an LBV stage. 


\subsection{WO stars}

Sander et al. also showed that the Galactic WO2 stars WR 142 and WR 102 have significantly different parameters from the WC stars. The WO positions in the HR diagram suggest that these stars could be close to or already in the stage of carbon burning which makes them interesting SN Ic candidates (Georgy et al. 2012; Yoon et al. 2012).

Tramper et al. presented VLT/X-Shooter spectroscopy of DR1, a WO3 star in the lowmetallicity galaxy IC 1613. A preliminary spectroscopic analyis using CMFGEN indicates a high temperature (of $\sim 150 \mathrm{kK}$ ) which is also supported by the very strong nebular He II emission. The oxygen abundance does not seem to be enhanced compared to values found for early-type WC stars, suggesting that the strong oxygen emission is likely a temperature effect, rather than being caused by an increased oxygen abundance.

\subsection{Rapidly rotating WR stars}

The WR rotation issue is especially relevant in view of the suggested link between rotating WR stars and long-duration GRBs. WR 2 (WN2) is a well known oddball. It is the most compact and the hottest $(\sim 140 \mathrm{kK})$ pop I WN star known in the Galaxy. It is also one of the best candidate for strange-mode pulsations (Glatzel et al. 1999), but they are not observed in photometric observations. The spectrum displays bowler-hat shaped emission lines, in contrast with the more normal Gaussian or even flat-top and triangular profiles of most other WR winds. Remarkably, Hamann et al. (2006) have analyzed WR 2 using their latest model-atmosphere code, and the model spectrum failed to reproduce its line-profiles, unless it was folded with a rotation curve near the break-up limit, i.e. $1900 \mathrm{~km} / \mathrm{s}$.

However, Chenè presented the polarized spectrum of WR 2, which shows no sign of wind asymmetry expected for such rapid rotation. Interestingly, WR 2 appears to display clumps that are moving in a similar fashion as in the optically thinner wind of WR 3 (Chené et al. 2008). Hence, the shape of WR 2 spectra line cannot be the result of extreme opacity either.

\subsection{Massive stars in the low metallicity galaxy IC1613}

Because low metallicity environments may favour the formation of massive and very massive stars, we switch our attention to extra-galactic properties. Herrero discussed the selection of massive star candidate stars in the low-Z galaxy IC 1613 from the catalog of Garcia et al.(2009). IC 1613 has a metallicity $\mathrm{Z}=0.13 \mathrm{Z}_{\odot}$ according to the analysis of B-supergiants by Bresolin et al. (2007), or $\mathrm{Z}=0.08-0.15 \mathrm{Z}_{\odot}$ according to several analyses of HII regions (see Herrero et al. 2012). Herrero et al. observed the selected stars with OSIRIS/GTC at a resolution $R=1000$ to determine their spectral types, as a previous step for a more detailed analysis. They were able to classify 12 new OB stars and confirmed one more known O-type star.

The spectra of the $\mathrm{O}$ stars were good enough for quantitative analysis (albeit with errors slightly larger than in typical analyses, see Repolust et al. 2004). Combining these results to those from the literature (Tramper et al. 2011; Herrero et al. 2012), Herrero presented the first effective temperature scale for sub-SMC metallicities. This temperature scale is slightly hotter than that derived for SMC stars from the data of Massey et al. (2009), Mokiem et al. (2007), and Trundle et al. (2007).

\subsection{Stellar abundances from massive stars in $M 33$}

With its distance less than a Mpc (Bonanos et al. 2006) and its favourable inclination angle, this makes M33 an ideal galaxy to study the chemical evolution in spiral galaxies. 
Not long ago, the only way to carry out detailed chemical analyses of nearby galaxies was through the quantitative studies of H II regions. However, the abundances derived from massive OB stars are the tracers of the present-day chemical composition, providing information that cannot be obtained H II regions (e.g. the silicon abundance). Moreover, a simultaneous characterization of the stellar parameters may address important aspects of their evolution, and in particular the role of environmental factors.

Castro presented the results of a spectroscopic survey in M 33 involving 59 supergiants with spectral types between B9 and O9, and a quantitative analysis according to the steps described by Castro et al. (2012). A thorough comparison between optical spectra and new FASTWIND (Puls et al. 2005) grids resulted in both stellar parameters and chemical composition. The parameters derived in conjunction with the evolutionary tracks of Brott et al. (2011) hints at the presence of evolved stars with masses in the range 15 and $50 \mathrm{M} \odot$. New routines for deriving the chemical abundances automatically through a process optimization showed an oxygen distribution along M 33 that is compatible with previous H II region studies (e.g. Rosolowsky \& Simon 2008).

\section{Mass loss mechanisms for VMS}

The evolution of Very Massive Stars is presumably dominated by mass loss, which thus needs to be understood both qualitatively and quantitatively. Joachim Puls reviewed different mass-loss mechanisms relevant in this context. Because of the high luminosities, only radiation-driven mass loss was considered, and time-dependence, rotation and magnetic fields were not accounted for.

\subsection{Theoretical considerations}

Basic considerations. The equation of motion for the transonic/supersonic regime of an expanding wind can be approximated by

$$
v\left(1-\frac{a^{2}}{v^{2}}\right) \frac{\mathrm{d} v}{\mathrm{~d} r} \approx g_{\text {grav }}(r)+g_{\mathrm{rad}}^{\mathrm{tot}}(r)=-\frac{G M}{r^{2}}(1-\Gamma(r)), \quad \Gamma(r)=\frac{\bar{\kappa}(r) L_{*}}{4 \pi G M c}=\frac{\bar{\kappa}(r)}{\sigma_{\mathrm{e}}} \Gamma_{\mathrm{e}}
$$

where all quantities have their usual meaning, $a$ is the isothermal sound speed, $g_{\mathrm{rad}}^{\text {tot }}$ is the total radiative acceleration (lines + continuum), $\bar{\kappa}$ is the flux-mean opacity per unit mass, and $\Gamma$ the corresponding Eddington parameter ( $\Gamma_{\mathrm{e}}$ w.r.t. electron scattering only). At the sonic point, $r_{s}, v=a$, and thus $g_{\text {rad }}^{\text {tot }}=-g_{\text {grav }}$ implying $\Gamma\left(r_{s}\right)=1$. To allow for an accelerating wind, $\left.\frac{\mathrm{d} v}{\mathrm{~d} r}\right|_{s}>0$, which then requires $\left.\frac{\mathrm{d} \bar{\kappa}}{\mathrm{d} r}\right|_{s}>0$ and $\Gamma(r)<1$ below and $\Gamma(r)>1$ above the sonic point, respectively.

Photon tiring limit. The mechanical luminosity of the wind at 'infinity' is given by

$$
L_{\text {wind }}=\dot{M}\left(\frac{v_{\infty}^{2}}{2}+\frac{G M}{R}\right)=\dot{M}\left(\frac{v_{\infty}^{2}}{2}+\frac{v_{\mathrm{esc}}^{2}}{2}\right) \quad \text { with } \quad v_{\mathrm{esc}}=\sqrt{\frac{2 G M}{R}},
$$

and the maximum mass-loss rate follows from the condition that $L_{\text {wind }}=L_{*}$ (when the star would become invisible): $\dot{M}_{\max }=2 L_{*} /\left(v_{\infty}^{2}+v_{\mathrm{esc}}{ }^{2}\right)$. Following (Owocki \& Gayley 1997), $\dot{M}_{\text {tir }}$ then is the maximum mass-loss rate when the wind just escapes the gravitational potential, with $v_{\infty} \rightarrow 0$, and is much larger than typical mass-loss rates from line-driven winds,

$$
\dot{M}_{\mathrm{tir}}=\frac{2 L_{*}}{v_{\mathrm{esc}}{ }^{2}}=0.032 \frac{M_{\odot}}{\mathrm{yr}} \frac{L_{*}}{10^{6} L_{\odot}} \frac{R}{R_{\odot}} \frac{M_{\odot}}{M}=0.0012 \frac{M_{\odot}}{\mathrm{yr}} \Gamma_{\mathrm{e}} \frac{R}{R_{\odot}} .
$$


Continuum driven winds. To drive a wind by pure continuum acceleration $\left(\Gamma=\Gamma^{\text {cont }}\right)$ when the photosphere is sub-Eddington $\left(\Gamma(r)<1\right.$ for $\left.r<r_{s}\right)$ requires substantial finetuning to reach and maintain $\Gamma^{\text {cont }}(r) \geqslant 1$ for $r \geqslant r_{s}$, and is rather unlikely. Anyhow, in such a situation $g_{\text {rad }}^{\text {cont }}$ is almost density-independent, and large mass-loss rates could be accelerated, only limited by photon tiring, which needs to be considered in the equation of motion (for details, see Owocki \& Gayley 1997).

Super-Eddington winds. If, on the other hand, the complete atmosphere is superEddington, $\Gamma(r)>1$, continuum driving (mostly due to electron-scattering) might become possible. When atmospheres approach or exceed the Eddington limit, non-radial instabilities arise making them inhomogeneous (clumpy). Photons on their way out avoid regions of enhanced density, and the medium becomes porous. In this case, the photospheric radiative acceleration decreases compared to an unclumped medium, leading to an effective Eddington parameter below unity. In the outer regions, where the clumps become optically thin due to expansion, porosity decreases and $\Gamma_{\mathrm{eff}}^{\mathrm{cont}} \rightarrow \Gamma(r)>1$. Thus, an accelerating wind can be initiated (Shaviv 2000, 2001a,b). (Owocki et al. 2004) expressed the effective opacity in terms of a 'porosity length', and showed that associated mass-loss rates can become substantial when this length is on the order of the pressure scale height, amounting to a few percent of the tiring limit. Invoking a power-law distributed porosity length, they showed that the 'observed' mass loss from the giant outburst of $\eta$ Car might be explained by this (metallicity-independent!) mechanism, and that $\dot{M}$ scales with $\dot{M} \propto \Gamma^{1 / \alpha_{p}-1}$, as long as the exponent of the power-law, $\alpha_{p}<1$ and $\Gamma>3 \ldots 4$.

Line-driven winds. The standard theory of line-driven winds (Castor et al. 1975 and later refinements) assumes that the continuum is still optically thin at the sonic point (valid for OB-stars, A-supergiants and LBVs in their quiet phase), and that $\Gamma^{\text {cont }}<1$ everywhere, with $\Gamma^{\text {cont }} \rightarrow \Gamma_{\mathrm{e}}$ for $r \geqslant r_{s}$. The radiative acceleration exerted on a shell of mass $\Delta m=4 \pi r^{2} \rho$ by one optically thick line can be expressed as

$$
g_{\mathrm{rad}}^{\text {one line }}=\frac{\Delta P}{\Delta t \Delta m} \propto \frac{L_{\nu} \nu}{4 \pi r^{2}} \frac{\mathrm{d} v}{\rho \mathrm{d} r},
$$

where $\Delta P$ is the transferred momentum, and the term $\mathrm{d} v$ arises because of the Dopplershift within $\mathrm{d} r$. Summing up over all lines using a line-strength distribution function and accounting for optical depth effects, the total line acceleration results in

$$
g_{\text {rad }}^{\text {all lines }} \propto N_{0} \frac{L_{*}}{4 \pi r^{2}}\left(\frac{\mathrm{d} v}{\rho \mathrm{d} r}\right)^{\alpha} \rightarrow N_{0} \frac{L_{*}}{4 \pi r^{2}}\left(\frac{4 \pi}{\dot{M}}\right)^{\alpha}\left(r^{2} v \frac{\mathrm{d} v}{\mathrm{~d} r}\right)^{\alpha},
$$

with $N_{0}$ the effective number of driving lines (depending on spectral type and metallicity), and $0<\alpha<1$ related to the slope of the line distribution function. Inserting this expression into the equation of motion and neglecting photon tiring, a unique (maximum) mass-loss rate can be calculated,

$$
\dot{M} \propto N_{0}^{1 / \alpha} L_{*}^{1 / \alpha}\left(M\left(1-\Gamma_{\mathrm{e}}\right)\right)^{1-1 / \alpha}=N_{0}^{1 / \alpha} L_{*}\left(\frac{\Gamma_{\mathrm{e}}}{1-\Gamma_{\mathrm{e}}}\right)^{1 / \alpha-1}=\left(\frac{N_{0} \Gamma_{\mathrm{e}}}{1-\Gamma_{\mathrm{e}}}\right)^{1 / \alpha} M\left(1-\Gamma_{\mathrm{e}}\right)
$$

which dramatically increases for $\Gamma_{\mathrm{e}} \rightarrow 1$ (in this case, photon-tiring needs to be accounted 


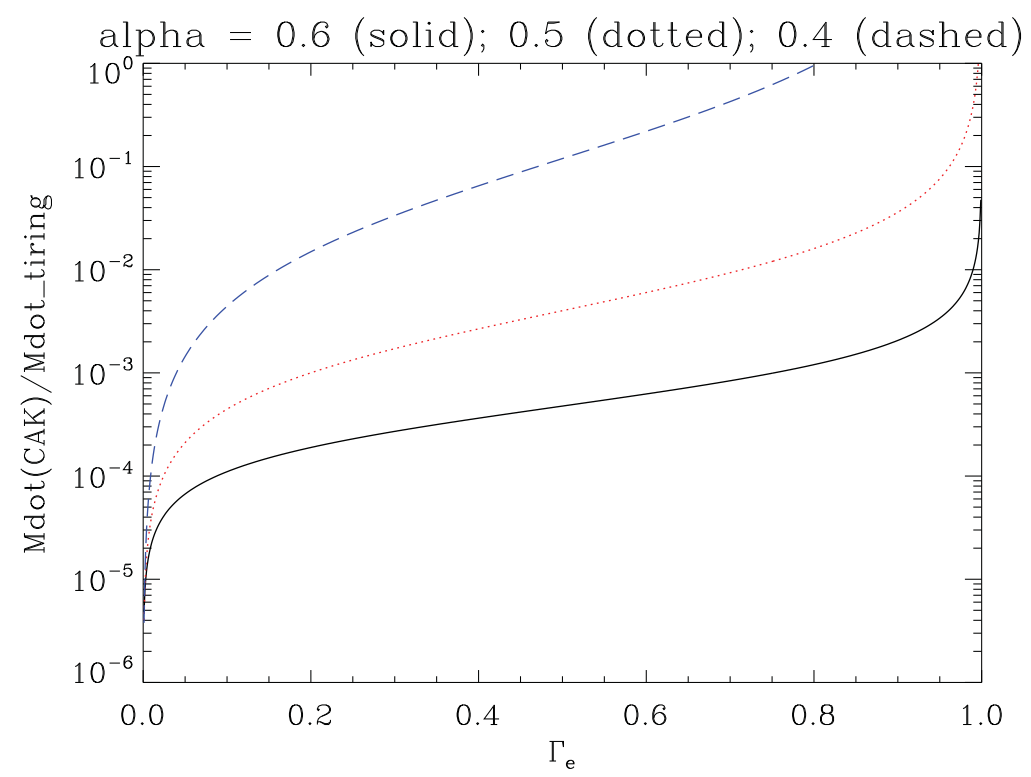

Figure 6. $\dot{M} / \dot{M}_{\text {tir }}$ for a typical O-star wind with $v_{\mathrm{esc}}=600 \mathrm{~km} \mathrm{~s}^{-1}$, as a function of $\Gamma_{\mathrm{e}}$ and $\alpha$.

for). On the other hand, the terminal velocity scales with

$$
v_{\infty} \propto v_{\mathrm{esc}}^{\mathrm{eff}}=\sqrt{\frac{2 G M\left(1-\Gamma_{\mathrm{e}}\right)}{R}} \rightarrow 0 \text { for } \Gamma_{\mathrm{e}} \rightarrow 1 .
$$

Figure 6 displays the ratio of $\dot{M} / \dot{M}_{\text {tir }}$ as a function of $\Gamma_{\mathrm{e}}$, and shows that this ratio is strongly sensitive to $\alpha$. For $\alpha=0.4$ (somewhat lower than the typical value for OB-stars), the tiring limit would already be reached at $\Gamma_{\mathrm{e}}=0.8$.

Optically thick winds. The large mass-loss rates from WR-stars, being typically a factor of 10 higher compared to OB-star winds at the same luminosity, cannot be explained by the standard theory from above. The observed terminal velocities (similar to OB-stars) can be reached only when line-overlap effects become efficient. In such dense winds, the ionization equilibrium decreases outwards, and photons on their way out can interact with lines from different ions, whilst any 'gaps' between lines (as present in OB-stars because of an almost frozen-in ionization) are closed (see Lucy \& Abbott 1993; Springmann 1997). The initiation of the mass loss, on the other hand, is supposed to rely on the condition that the winds are already optically thick at the sonic point, and that the (quasi-static) photospheric line acceleration due to the iron peak opacities around $150 \mathrm{kK}$ (for WNEs) or $40 \mathrm{kK}$ (for WNLs) is sufficient to overcome gravity (Nugis \& Lamers 2002). (Gräfener \& Hamann 2005, 2008) calculated self-consistent models for WNEs and WNLs, and showed that this mechanism actually allows for large $\dot{M}$, where the major prerequisite is a high $\Gamma$. Such optically thick winds might be present also in VMS (Vink et al. 2011 and these proceedings), although (Pauldrach et al. 2012) argue that VMS winds might remain optically thin.

\subsection{Monte Carlo mass-loss rates}

Vink discussed three relatively new aspects concerning mass-loss calculations from the Monte Carlo method (Abbott \& Lucy 1985) - as previously used to predict $\dot{M}$ for canonical OB-type stars (e.g. Vink et al. 2000). The first one concerned the wind dynamics. Until 
2008, the methodology was semi-empirical, as a velocity law was assumed that reached a certain empirical $v_{\infty}$. Müller \& Vink (2008) suggested a line-force parametrization that explicitly depends on radius (rather than the velocity gradient, as in CAK theory), and predicted $v_{\infty}$ values in reasonable agreement with observations. Muijres et al. (2012) tested the Müller \& Vink approach, and as both methods gave similar results, it was used in the following.

Secondly, a new parameter space was probed, i.e. that of the VMS. Vink et al. (2011) $\dot{M}$ predictions show a kink in the $\dot{M}$ - $\Gamma$ relation. For "low" $\Gamma$ optically-thin O-star winds, the $\dot{M} \propto \Gamma^{x}$ relation is shallow, with $x \simeq 2$, whilst there is a steepening at high $\Gamma$, with $x \simeq 5$. At high $\Gamma$ the objects show optically thick WR-like winds, with optical depths and wind efficiencies above unity. Gräfener et al. (2011) provided empirical evidence for such a steep exponent $(x \simeq 5)$, but there are still issues with the predictions of absolute $v_{\infty}$ values in this high $\Gamma$ range. Critical comparisons between observations and theory are underway by Bestenlehner et al. in the context of the VFTS survey (Evans et al. 2011)

In another study Vink \& Gräfener (2012) calibrated wind mass-loss rates using an analytic method to find that the wind efficiency number equals unity right at the transition point between optically thin and thick winds: $\eta=\tau=1$. Application of this relation to the most massive stars in the Arches cluster suggests there is little room for additional mass loss during e.g. LBV eruptions, and current wisdom would suggest that PSN explosions are unlikely, unless one were to move to lower $Z$ galaxies (e.g. Langer et al. 2007, Yoshida \& Umeda 2011, Yusof et al. in prep.).

\subsection{Alternative mass loss: eruptions and mass transfer}

Solar metallicity VMS likely evaporate as the result of stellar wind mass loss. However, alternative mass loss may also be important, especially for the lower initial mass and subsolar metallicity ranges. Furthermore, we know Eta Car analogs and supernova impostors exist in external galaxies (e.g. Van Dyck et al. 2005, Pastorello et al. 2010, Kochanek et al. 2012), but quantitative estimates on the integrated amount of such eruptive mass loss are hard to obtain as both the eruption frequency, and the amounts of mass lost per eruption span a wide range with LBV nebular mass estimates varying from $\sim 0.1 M_{\odot}$ in P Cygni to $\sim 10 M_{\odot}$ in Eta Car (Smith \& Owocki 2006).

The energies required to produce such giant mass eruptions are very high $\left(\sim 10^{50} \mathrm{erg}\right)$, and their energy source is unknown. Soker (2004) discussed that the energy and angular momentum required for Eta Car's great eruption cannot be explained with a single-star scenario.

There is a growing amount of evidence that the most massive stars are oftentimes found in binary systems, and binary evolution with mass loss is pursued by many groups around the globe (Vanbeveren 1998; Eldridge et al. 2008). What has become particularly clear from recent spectroscopic radial velocity surveys (e.g. Sana et al. 2012) is that there is a particularly large number of short-period binaries, which might merge still during core $\mathrm{H}$ burning, and subsequently evolve as seemingly single stars. For these reasons one of the most famous recent phrases in the massive star community has been "binary stars might actually be the best single stars" (de Mink et al. 2011). However, also after the main sequence, there are still many physical processes involving mass loss through Roche lobe overflow (see e.g. Langer 2012) and common envelope evolution (Ivanova et al. 2012), which remain as yet ill-understood. 


\subsection{Mass-loss diagnostics}

The traditional ways of determining mass-loss rates of (very) massive stars involve recombination lines (such as $\mathrm{H} \alpha$ and He II 4686), as well as radio and sub-mm continuum measurements that measure the amount of free-free emission (Wright \& Barlow 1975, Lamers \& Cassinelli 1999). Especially the free-free method may be used in the near future with new facilities such as ALMA coming online. The drawback of the above diagnostics is that they depend on an uncertain amount of wind clumping (Puls et al. 2008). The unsaturated resonance lines of trace elements, such as $\mathrm{P} v$, located in the far UV, has been considered a more accurate tool for mass-loss diagnostics (e.g. Fullerton et al. 2006), because their formation depends linearly on density such that inhomogeneities average out. However, it was shown by Oskinova et al. (2007) that the formation of resonance lines is also affected by wind clumping, if the line opacity makes the individual clumps optically thick. Neglecting this effect may lead to underestimations of the true $\dot{M}$ (see also Sundqvist et al. 2010).

For these reasons it is important to (i) gain a greater understanding of both the physics and the diagnostics of wind clumping throughout the stellar atmosphere and wind, ideally as a function of radial distance, and (ii) to develop diagnostics that are not dependent on wind clumping.

\subsubsection{X-ray diagnostics for VMS}

Massive stars of most (but not all) spectral types are sources of X-ray emission. In single stars, the X-rays most likely originate in the gas heated by the strong shocks resulting from the line-driven instability of stellar winds (e.g. Lucy 1980, Owocki et al. 1988). Therefore, the properties of X-ray emission are sensitive to the wind driving mechanism. Because of the proximity of VMSs to the Eddington limit, the details of wind driving and line-driving instability growth may be different from lower mass massive stars.

The X-ray luminosity of VMS stars is challenging to predict. The X-ray luminosity of Galactic OB stars follows the trend $L_{\mathrm{X}} \propto 10^{-7} L_{\mathrm{bol}}$. While some binary $\mathrm{O}$ stars with colliding winds have X-ray luminosity significantly higher than $10^{-7} L_{\mathrm{bol}}$, the majority of O star binaries follow this correlation as well (Oskinova 2005, Naze 2009). In some cases, the binary $\mathrm{O}$ stars have X-ray luminosity significantly lower than the expected for a single star of similar spectral type. Oskinova produced a diagram showing the dependence of the X-ray luminosity in binary $\mathrm{O}$ stars on the period. No correlation was seen, and the short period binaries can have low X-ray luminosity.

Oskinova concluded that a low X-ray luminosity cannot serve as a robust argument against a binary nature of an $\mathrm{O}$ star. In other words, a binary luminous VMS could potentially have a low $L_{\mathrm{X}}$. However, the story for the WR-like VMS binaries (with strong winds) might be different from the weaker-winded O-star binaries.

\subsubsection{A new wind measurement approach using X-rays from colliding wind binaries}

Sugagawa presented Suzaku observations of the WR binary WR 140, taken at four different times around periastron passage in 2009 January. The X-ray spectra changed in shape and flux at each phase. As periastron approached, the column density of the low-energy absorption increased, indicating that the emission from the wind-wind collision plasma was absorbed by the dense WR wind. The luminosity of the dominant hot component from the wind-wind collision is not inversely proportional to the (variable) distance between the two stars. In the case of the mass-loss ratio $\dot{M}_{\mathrm{O}} / \dot{M}_{\mathrm{WR}}=0.04$, Sugagawa could explain this discrepancy if the O-star wind collides with the WR wind before it has reached its terminal velocity, leading to a reduction in its wind momentum flux. 
Sugagawa presented these mass-loss rates, which were calculated using the absorptions and variations of the spectra (Sugawara et al. 2012, submitted).

Daminelli showed that the He II 4686 line in Eta Carinae displays two peaks before periastron in good correlation with the X-ray intensity. There even is a third peak, in close coincidence with periastron, which is anti-correlated with X-rays intensity. This may be interpreted as a collapse in the wind-wind collision structure, when most of the energy escapes in the extreme UV, which would be possible if the eccentricity is larger than e $>0.9$.

What is clear is that the approach presented by Sugagawa using X-ray observations is applicable to other massive CWBs with elliptical orbits. In addition, unexpected X-ray brightening of very massive CWBs (such as WR 21a) may be helpful for understanding VMS mass loss.

\subsubsection{Mass loss at very low metallicity}

Whilst there are still several uncertainties in our empirical knowledge of Solar metallicity mass loss, rates at low $Z$ may provide additional constraints on the driving mechanisms. In this context, Tramper presented the results of a quantitative spectroscopic analysis of VLT/X-Shooter observations of six O-type stars in the low-metallicity galaxies IC 1613, WLM and NGC 3109 (Tramper et al., 2011; Herrero et al. 2011, but see also Herrero et al. 2012). The obtained stellar and wind parameters can be used to probe the mass loss versus metallicity dependence at metallicities below that of the SMC. Tramper et al. compared their derived mass-loss rates with the empirical results from Mokiem et al. (2007) for the Galaxy, LMC and SMC, and with the theoretical prediction from Vink et al. (2001), and argued that the mass-loss rates appear to be higher than expected.

It is clear that the analysis of a larger sample of stars at sub-SMC metallicities is needed to confirm or disprove these results.

\section{VMS Evolution and Fate}

Alexander Heger (with Woosley \& Chen) presented the fourth review talk on (very) massive star evolution. An introduction to massive star evolution can be found in (Woosley et al. 2002; Heger 2012). One of the most exciting questions concerning massive stars is how they will die, which of them will explode, and how. A range of outcomes is possible in terms of observational signatures, but the key question is how massive the star is at the time of death. Therefore, according to Heger understanding the mass-loss rates of massive stars is one of the key uncertainties that needs to be resolved.

Other stumbling blocks along the way involve rotation and binary evolution. For the sake of simplicity, Heger only discussed single stars. However, it is clear that some close binaries will merge early during their evolution, already during central hydrogen burning. These would likely evolve in a fashion similar to that of single stars - though likely rapidly rotating. Rotation however is a second parameter that makes the picture more complex. So for the sake of simplicity Heger dealt "just" with single stars and deferred the reader to the rotation works of (Yoon et al. 2006, 2012). In some aspects, rapidly rotating single stars may evolve in a fashion similar to non-rotating stars of a different (usually higher) mass, maybe if mass-loss rates are varied (artificially) similar results can be obtained.

For the final death, the explosion mechanism and magnitude, knowing the size of the (helium) core is the key ingredient. Heger discussed outcomes in terms of initial masses for Pop III stars where it was also assumed there is no mass loss by stellar winds, taking 
the key uncertainty out of the equation, and hence allows one to draw a clearer picture - though obviously not realistic. However, it allows one to understand and formulate the possible outcomes.

Supernovae from lower mass massive stars generally produce neutron stars within the 'classical' core collapse SN scenario. For higher masses, again really meaning for higher mass cores at the end of evolution, one eventually has to deal with core structures that no longer allow for an efficient explosion and expect the star to collapse to a black hole. Depending on the rotation of the star, a GRB may result. Again, this may require binary stars or rapidly rotating single stars, but here the focus is on the dependence on star/core mass for possible outcomes. A recent review on such possible outcomes can also be found in (Woosley \& Heger 2012).

When the mass of the star exceeds some $90-100 M_{\odot}\left(\sim 42 M_{\odot}\right.$ He core $)$, pulsational pair instability (Puls-PSN) can occur. These are violent nuclear-powered pulsations during the final burning stages, usually powered by oxygen or silicon burning. They may produce as much energy as a SN, or more. However, it could also be significantly less. Heger discussed this in more detail (see below). After the pulsing is over and the star forms an iron core, the fate should be similar to the more massive stars discussed above. For higher initial masses, above some $140 M_{\odot}\left(65 M_{\odot}\right.$ He core $)$ the first pulse is already powerful enough to disrupt the star and a pair instability (PSN) results; for even higher initial masses, above some $260 M_{\odot}\left(133 M_{\odot}\right.$ He core $)$ it is expected that the star collapses to a black hole. A brief discussion on what could happen in that case may be found in (Fryer et al. 2001).

One of the striking problems with the PSN theory is that they produce a unique chemical signature - at least for the non-rotating primordial stars. Simulations of the formation of the first stars suggest that they should have been rather massive (e.g., Abel et al. 2002), hence making PSN, and their nucleosynthesis products should have been incorporated into the next generation of stars, but to date, no stars with such nucleosynthesis pattern have been found.

\subsection{Pulsational pair instability (Puls-PSN)}

Figure 7 depicts the dynamics of a Puls-PSN simulation in 1D: after the pulse there is a ring-down phase in which radiative dampening by neutrino losses brings the star back into hydrostatic equilibrium. It is noted that the core of the star is much more extended after the pulse than it was before (the reader may follow convective boundaries as a reference). In other words, the energy deposited by the burning during the pulse leads to an expansion and cooling of the stars - as we know from textbooks, stars have a negative (gravothermal) heat capacity. But in order to get ready for the next pulse, it needs to get hot enough, and the energy needs to be lost again. The more energetic, the cooler the core of the star will be. But now the end stages of massive stars are dominated by neutrino losses, and these are known to depend steeply with temperature. Therefore, the cooler the star, the longer it takes to cool.

In extreme cases of rather powerful explosions, the temperature could drop enough such that the core has to cool on the classical Kelvin-Helmholtz time for radiative losses from the surface, as it has become so cool that neutrinos are no longer efficient. This can increase the recurrence time by several orders of magnitude. In summary, we generally expect that more powerful pulses result in longer delay times, whereas weak pulses result in short delay times for the next pulse. The recurrence times can range from hours to more than 10,000 yr, and the energies from $0.0001 \mathrm{~B}$ to several $\mathrm{B}\left(1 \mathrm{~B}=1 \times 10^{51} \mathrm{erg}\right)$. 


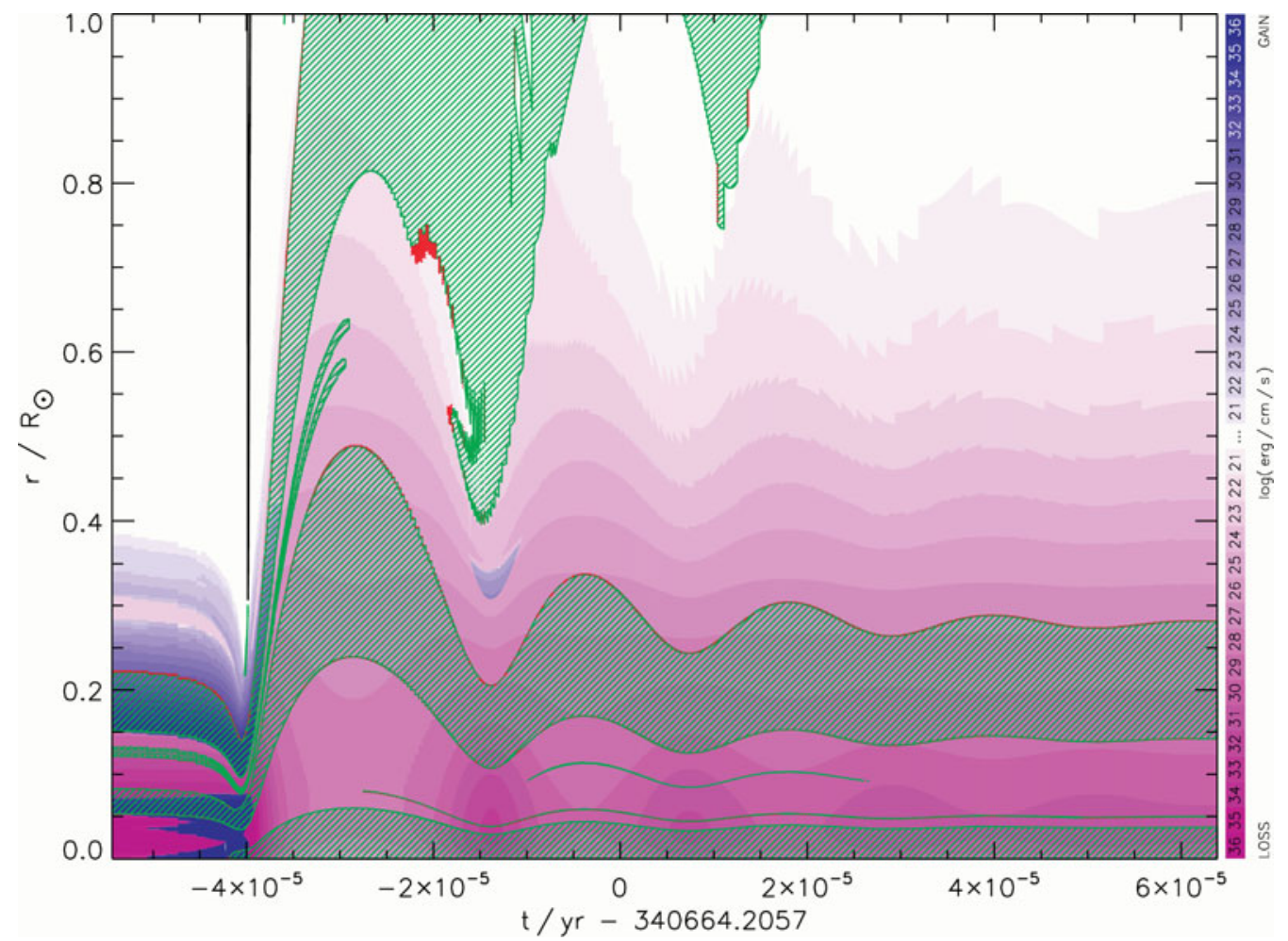

Figure 7. Energy loss (purple), energy generation (blue), and convection (green hatching) during a pulsational pair instability pulse of a $100 M_{\odot}$ star. The $x$-axis indicates the time, $t$, in years since the beginning of helium burning, the $y$-axis shows the radius coordinate from the center of the star in solar radii.

Why is all this relevant? Because we wish to understand whether some giant eruption LBVs could be the result of Puls-PSN. In the case of Eta Car we dealt with a fairly weak explosion, as some of the $\mathrm{H}$ remained bounded to the star, so we would have expected the next pulse to happen soon after Eta Car's giant eruption. However, this was not observed, or so it appears. Therefore, at this point it seems unlikely that Eta Car's giant eruption was a Puls-PSN event.

\subsection{Stellar Envelope Inflation}

Alternative explanations for LBV outbursts and eruptions have been proposed over the years (see Humphreys \& Davidson 1994; Vink 2009). Gräfener et al. (2012) proposed the possibility that envelope inflation near the Eddington limit may play a key role in explaining the radius increases during S Dor cycles. The peculiar structure of inflated envelopes, with an almost void region beneath a dense shell could mean that many in reality compact stars are hidden below inflated envelopes, displaying much lower effective temperatures (see also Ishii et al. 1999; Petrovic et al. 2006).

During the JD, Gräfener discussed the inflation effect for WR stars, whose observed radii are up to an order of magnitude larger than predicted by theory. Based on a new analytical formalism, he described the radial inflation as a function of a dimensionless parameter $W$, which largely depends on the topology of the Fe-opacity peak, i.e., on material properties. For $W>1$, an instability limit is found for which the stellar envelope becomes gravitationally unbound, i.e. there no longer exists a static solution. Within this 
framework one may also be able to explain the S Doradus-type instabilities for LBVs like AG Car (discussed by Groh during the meeting). Moreover, due to the additional effect of sub-photospheric clumping, it may be possible to bring the observed WR radii in agreement with theory (see Sander et al. results discussed earlier).

It should be noted that stellar effective temperatures in the upper HR diagram may be strongly affected by the inflation effect. This may have particularly strong effects on the evolved massive LBV and WR stars just prior to their final collapse, as the progenitors of supernovae (SNe) Ibc, SNe II, and long-duration GRBs.

\subsection{D Simulations of Thermonuclear Supernovae from VMS}

Ke-Jung Chen (with Heger \& Woosley) presented results from numerical simulations of the demise of VMS with initial masses between $140 M_{\odot}$ and $250 M_{\odot}$ that can die as powerful PSN explosions. Chen et al. used CASTRO, a new multidimensional radiationhydrodynamics code, to study the evolution of PSNe. The 3D simulations start with the collapse phase and follow the explosion until the shock breaks out from the stellar surface. Unlike the iron-core collapse SNe, PSNe are powered by thermonuclear runaway without leaving compact remnants. Much $\mathrm{Ni}$ is forged, up to $30 M_{\odot}$, and its decay energy powers the PSN luminosity for several months. During the explosion, the emergent fluid instabilities cause the mixing of PSN ejecta, and the amount of mixing is related to PSN progenitors. The red supergiant progenitors demonstrate strong mixing, altering the spectrum and light curves.

\section{Implications}

\subsection{Population synthesis models}

The implications for the existence of VMS may be far-reaching, as VMS may dominate both the kinetic wind energy input and the ionizing radiation in the Universe. Because of higher temperatures at lower metallicities, VMS may be increasingly UV bright. However, their higher luminosities might imply higher mass-loss rates and terminal wind velocities, which would account for an increased kinetic wind energy.

Voss presented his recent population models (Voss et al. 2009) which follow both the energy - in the form of kinetic wind energy as well as radiation - and the ejection of radio-active isotopes (such as ${ }^{26} \mathrm{Al}$ ) simultaneously. Below $120 M_{\odot}$, stellar evolution calculations predict a strong increase in the ejected mass of ${ }^{26} \mathrm{Al}$ with stellar mass. The ejection of ${ }^{26} \mathrm{Al}$ from stars above this limit has not been studied in detail, but as the mass-loss increases dramatically towards higher masses, it is reasonable to expect the ${ }^{26} \mathrm{Al}$ to do the same. The short evolutionary timescale of VMSs mean that all the ${ }^{26} \mathrm{Al}$ is ejected 2-4 Myr after the star-forming episode, and if present, the VMSs will dominate the signal for 2-5 Myr populations, but due to the decay their signal will be negligible for older populations. Comparing the ${ }^{26} \mathrm{Al}$ signal from 2-5 Myr massive open clusters to their 5-10 Myr counterparts is therefore a promising way to probe the evolution of VMSs (Voss et al. 2010; 2012).

\subsection{Wolf-Rayet Stars in the Extraordinary Star Cluster NGC 3125-A1}

The powerful radiative and mechanical feedback from very massive stars $\left(\geqslant 100 \mathrm{M}_{\odot}\right)$ shape the evolution of star-forming galaxies and their environments. Nearby galaxies 
$(\lesssim 10 \mathrm{Mpc})$ provide excellent laboratories for studying populations of such stars in sufficient detail in a variety of astrophysical environments.

Aida Wofford (with Leitherer and Chandar) studied the massive star populations of clusters A1, A2, B1, and B2 in blue compact dwarf galaxy NGC 3125, which is located 11.5 Mpc away and has an LMC-like metallicity. It is unclear from past studies if cluster A1 hosts an extreme population of WR stars. In addition, the WR star populations of the other clusters are not well characterized. Wofford et al. obtained HST/STIS 1200-9000 $\AA$ spectra of these four clusters, and higher resolution HST/COS 1200-1450 A spectra of cluster A1, on which Wofford focused. The STIS spectrum of this cluster shows that the equivalent width of He II $\lambda 1640$ is three times the mean of local starburst galaxies (Chandar et al. 2004) and three times the value of the strongest Lyman Break Galaxy (Erb et al. 2010). This suggests that A1 must have a large fraction of WR stars relative to the number of $\mathrm{O}$ stars. Either A1 has a top heavy IMF or it contains a few massive stars with very strong winds. The COS spectrum of A1 shows the strongest $\mathrm{OV}+$ Fe V absorption feature at $1371 \AA$ from a starburst in the local universe. The O V line originates in the most massive stars and is sensitive to clumping in the stellar wind. The analysis of the $\mathrm{OV}+\mathrm{FeV}$ using CMFGEN stellar atmosphere models is underway.

\subsection{Nebular He II 4686 emission: an indirect tracer of massive stars at low metallicities}

Shirazi (with Brinchmann) presented a carefully selected sample of 189 star-forming galaxies with strong nebular HeII 4686 emissions in Sloan Digital Sky Survey Data (SDSS) Release 7. They used this sample to investigate the origin of this high ionization line in star-forming galaxies where the ionizing continuum almost certainly arises from massive stars. The current stellar population models can predict He II 4686 emission only for instantaneous bursts of $20 \%$ solar metallicity or higher, and only for ages of 4-5 Myr, the period when the extreme-ultraviolet continuum is dominated by emission from WR stars.

Shirazi \& Brinchmann find however that 83 of the star-forming galaxies $(70 \%$ at oxygen-abundance lower than 8.2) of their sample do not have WR features in their spectra despite showing strong nebular He II 4686 emission. Nevertheless, at higher metallicities He II is always seen with WR features. Shirazi went on to show that the stacked spectra of the non-WR HeII emitters do not show WR features either, which suggests that the non-detection of WR features in these galaxies is not due to low signal-to-noise data, i.e. it is probably real.

Shirazi proposed that a possible explanation for the discrepancy between the model predictions and the observed data at very low redshifts could be the result of a spatial offset between the location of the WR stars and the region where the He II emission arises from. Alternatively, as the non-WR HeII emitters appear to be preferentially present in younger starbursts, (quasi)-chemically homogeneous stellar evolution could provide a possible explanation, as this may lead to higher stellar temperatures, and perhaps result in an elevated He II emission even for main-sequence O stars.

Shirazi \& Brinchmann are currently attempting to disentangle these explanations by analyzing higher signal-to-noise spectra of a sub-sample of these galaxies that were followed up with the WHT.

\subsection{Very Massive Stars in I Zw 18}

$\mathrm{IZw} 18$ is a blue compact dwarf (BCD) galaxy with the lowest metallicity known (at 1/30 $-1 / 50$ the solar value), and is therefore thought to be the best local galaxy template to 
galaxies at high redshift. Although IZw 18 is 15-19 Mpc away, Hubble/STIS imagery resolves stars in the galaxy.

Heap showed that UV color-magnitudes diagrams indicate that the most massive stars in the northwest cluster of IZw 18 are as massive as $150 M_{\odot}$. Heap also showed that Hubble/COS far-UV spectra reveal that the mass-loss rates from stars in the NW cluster must be very low, as only the N V 1240 doublet has a P Cygni profile, and it is very weak. The C IV 1549 doublet is resolved with an edge velocity of only $\sim 250 \mathrm{~km} / \mathrm{s}$. The emission component of the CIV doublet is quite possibly of nebular origin. Most of the 12 most luminous stars are bluer than ZAMS models, suggesting that the evolution of the most massive stars is affected by rapid rotation. A comparison of the observed CMD and FUV spectra with new evolutionary models including rotation will yield valuable information about chemical enrichment of the ISM, injected energy via ionizing radiation, and types of SN explosions in the NW cluster of I Zw 18.

\section{Final words}

One of the key science goals for the James Webb Space Telescope (JWST) is going to be the identification of the first galaxies with Pop III stars. These objects may have been very massive (up to $1000 M_{\odot}$ ). At this epoch black hole formation may have been more common than at the current time involving solar metallicities. The first couple of stellar generations may also have been responsible for the reionization of the Universe: an important cosmological epoch that is soon to be probed via the $21 \mathrm{~cm}$ line with instruments such as LOFAR.

With the E-ELT, individual (very) massive stars may be observed out to the Virgo cluster of galaxies in the Hubble flow, at ever larger distances, and for an increasing range of metallicities. Basic insights into mass loss at very low $Z$ involve issues such as the selfenrichment of metals through mixing (rotation, overshooting, magnetic fields, binarity) and mass loss in close proximity to the Eddington limit, which does not necessarily require metals, if the winds are continuum driven. We also need to consider the potential massloss-Z-dependence for the angular momentum evolution and the quest for pinpointing the progenitor stars of long-duration GRBs.

The fate of massive stars is important for our understanding of the chemical enrichment of the Universe. Whilst the deaths of stars up to $15 M_{\odot}$ now seem to be pretty well known; those of VMS up to $300 M_{\odot}$ are as yet a complete mystery. Their fates may involve PSN, Puls-PSN, or just normal hydrogen-poor SNe Ibc, either with our without an accompanying GRB. Key unknown aspects in this quest involve the strength and geometry of the progenitor stellar wind. Equatorial winds would remove angular momentum during evolution, whilst spherical winds would not. Linear spectropolarimetry should become a particularly powerful tool to study the geometry of winds and disks during the evolution of (very) massive stars towards explosion.

Similar arguments can be provided for the evolution of rotation rates during star formation. T Tauri and Herbig Ae/Be stars are the optically visible PMS up to $\sim 15 M_{\odot}$, and the formation of such objects is thought to proceed via disks. The latest observations and theoretical developments seem to suggest that ever more massive stars may form in similar fashions, i.e. via disk accretion, but before such far-reaching conclusions can be drawn, large stellar samples over the full IMF are needed. It is particularly important to explore the near IR part, in order to diminish the complications by dust extinction.

GAIA is widely expected to provide key information about the massive star formation history of our own Milky Way. This will involve issues of stellar dynamics, cluster 
formation, the role of massive star binarity, and the physical links between massive stars and their lower mass ( $\mathrm{T}$ Tauri) siblings.

Once we understand the upper IMF and the rotational/multiplicity properties of massive stars, we can concentrate on the evolution of the mass loss and rotation properties of VMS. Which objects could make PSNe and which ones might produce GRBs? May these transient phenomena become star-formation tracers at high redshift? If their luminosity functions could be mapped with respect to their redshifts, individual VMS may even allow astronomers to constrain galaxy formation models.

The future of VMS formation and evolution looks bright!

\section{Acknowledgements}

JSV would like to the the Royal Astronomical Society (RAS), the IAU, the UK Science and Technologies Facility Council (STFC) as well as the Northern Ireland Department of Culture Arts and Leisure (DCAL) for financial support.

\section{References}

Aarseth, S. J. 2003, Gravitational N-Body Simulations, by Sverre J. Aarseth, pp. 430, ISBN 0521432723, Cambridge University Press

Abbott, D. C. \& Lucy, L. B. 1985, ApJ, 288, 679

Abel, T., Bryan, G. L., \& Norman, M. L. 2002, Science, 295, 93

Andersen, M., Zinnecker, H., Moneti, A., et al. 2009, ApJ, 707, 1347

Barniske, A., Oskinova, L. M., \& Hamann, W.-R. 2008, A\& A, 486, 971

Banerjee, S., Kroupa, P., \& Oh, S., 2012a, MNRAS, 426, 1416

Banerjee, S., Kroupa, P., \& Oh, S., 2012b, ApJ, 746, 15

Bate, M. R. 2012, MNRAS, 419, 3115

Baumgardt, H. \& Klessen, R. S. 2011, MNRAS, 413, 1810

Belkus, H., Van Bever, J., \& Vanbeveren, D. 2007, ApJ, 659, 1576

Bestenlehner, J. M., Vink, J. S., Gräfener, G., et al. 2011, A\&A, 530, L14

Beuther, H., Sridharan, T. K., \& Saito, M. 2005, ApJ, 634, L185

Blandford, R. D. \& Payne, D. G. 1982, MNRAS, 199, 883

Bonanos, A. Z., Stanek, K. Z., Udalski, A., et al. 2004, ApJ, 611, L33

Bonanos, A. Z., Stanek, K. Z., Kudritzki, R. P., et al. 2006, ApJ , 652, 313

Bonnell, I. A., Bate, M. R., \& Zinnecker, H. 1998, MNRAS, 298, 93

Bontemps, S., Motte, F., Csengeri, T., \& Schneider, N. 2010, A\&AA, 524, A18+

Bresolin, F., Urbaneja, M. A., Gieren, W., Pietrzynski, G., \& Kudritzki, R.-P. 2007, ApJ 671, 2028

Bromm, V., Coppi, P. S., \& Larson, R. B. 1999, ApJ, 527, L5

Brott, I., de Mink, S. E., Cantiello, M., et al. 2011, A\&A A, 530, A115+

Brown, A. 2001, Astronomische Nachrichten, 322, 43

Campbell, M. A., Evans, C. J., Mackey, A. D., et al. 2010, MNRAS, 405, 421

Cassinelli, J. P., Mathis, J. S., \& Savage, B. D. 1981, Science, 212, 1497

Castor, J. I., Abbott, D. C., \& Klein, R. I. 1975, ApJ, 195, 157

Castro, N., Urbaneja, M. A., Herrero, A., et al. 2012, A\& $A$, 542, A79

Chandar, R., Leitherer, C., \& Tremonti, C. A. 2004, ApJ, 604, 153

Chatzopoulos, E. \& Wheeler, J. C. 2012, ApJ, 760, 154

Chené, A.-N., Moffat, A. F. J., \& Crowther, P. A., 2008, cihw.conf, 163

Clarkson, W. I., Ghez, A. M., Morris, M. R., et al. 2012, ApJ, 751, 132

Cooke, J., Sullivan, M., Gal-Yam, A., et al. 2012, Nature, 491, 228

Cucchiara, A., Cenko, S. B., Bloom, J. S., et al. 2011, ApJ, 743, 154

Commerçon, B., Teyssier, R., Audit, E., Hennebelle, P., \& Chabrier, G. 2011, A\& A, 529, A35+

Crowther, P. A., Schnurr, O., Hirschi, R., et al. 2010, MNRAS, 408, 731

Cunningham, A. J., Klein, R. I., Krumholz, M. R., \& McKee, C. F. 2011, ApJ, 740, 107 
Eldridge, J. J., Izzard, R. G., \& Tout, C. A. 2008, MNRAS, 384, 1109

Ellerbroek, L. E., Kaper, L., Bik, A., et al. 2011, ApJ, 732, L9

Erb, D. K., Pettini, M., Shapley, A. E., et al. 2010, ApJ, 719, 1168

Evans, C. J., Taylor, W. D., Hénault-Brunet, V., et al. 2011, A\&AA, 530, A108

Figer, D. F. 2005, Nature, 434, 192

Fryer, C. L., Woosley, S. E., \& Heger, A. 2001, ApJ, 550, 372

Fujii, M. S. \& Portegies Zwart, S. 2012, arXiv:1210.3732

Fullerton, A. W., Massa, D. L., \& Prinja, R. K. 2006, ApJ, 637, 1025

Gal-Yam, A., Leonard, D. C., Fox, D. B., et al. 2007, ApJ, 656, 372

Gal-Yam, A., Mazzali, P., Ofek, E. O., et al. 2009, Nature, 462, 624

Garcia, M., Herrero, A., Vicente, B., Castro, N., Corral, L. J., Rosenberg, A., \& Monelli, M. 2009, A\&SA 502, 1015

Georgy, C., Ekström, S., Meynet, G., et al. 2012, A\&A, 542, A29

Glatzel, W., Kiriakidis, M., Chernigovskij, S., \& Fricke, K. J. 1999, MNRAS, 303, 116

Glebbeek, E., Gaburov, E., de Mink, S. E., Pols, O. R., \& Portegies Zwart, S. F. 2009, A\&A, 497,255

Gräfener, G. \& Hamann, W.-R. 2005, A\&A, 432, 633

Gräfener, G. \& Hamann, W.-R. 2008, A\& $A$, 482, 945

Gräfener, G., Vink, J. S., de Koter, A., \& Langer, N. 2011, A\&̊A, 535, A56

Gräfener, G., Owocki, S. P., \& Vink, J. S. 2012, A\&A, 538, A40

Groh, J. H. \& Vink, J. S. 2011, A\&̧A, 531, L10

Groh, J. H., Hillier, D. J., Damineli, A., et al. 2009, ApJ, 698, 1698

Gürkan, M. A., Freitag, M., \& Rasio, F. A. 2004, ApJ, 604, 632

Hamann, W.-R., Gräfener, G., \& Liermann, A. 2006, A\&A, 457, 1015

Heger, A. 2012, in Astrophysics and Space Science Library, Vol. 384, Astrophysics and Space Science Library, ed. K. Davidson \& R. M. Humphreys, 299

Hennebelle, P., Commerçon, B., Joos, M., et al. 2011, A\& A, 528, A72+

Herrero, A., Garcia, M., Uytterhoeven, K., et al. 2011, IAU Symposium, 272, 292

Herrero, A., Garcia, M., Puls, J., Uytterhoeven, K., Najarro, F., Lennon, D. J., \& RiveroGonzlez, J. G. 2012, A\&3A 543, A85

Hillier, D. J. \& Miller, D. L. 1998, ApJ, 496, 407

Hoffmeister, V. H., Chini, R., Scheyda, C. M., et al. 2008, ApJ, 686, 310

Hosokawa, T., Yorke, H. W., \& Omukai, K. 2010, ApJ, 721, 478

Humphreys, R. M. \& Davidson, K. 1994, PASP 106, 1025

Ishii, M., Ueno, M., \& Kato, M. 1999, PASJ 51, 417

Ivanova, N., Justham, S., Chen, X., et al. 2012, arXiv:1209.4302

Jacquet, E. \& Krumholz, M. R. 2011, ApJ, 730, 116

Jijina, J. \& Adams, F. C. 1996, ApJ, 462, 874

Koen, C. 2006, MNRAS, 365, 590

Kotak, R. \& Vink, J. S. 2006, $A \& A$, 460, L5

Kratter, K. M. \& Matzner, C. D. 2006, MNRAS, 373, 1563

Kratter, K. M., Matzner, C. D., \& Krumholz, M. R. 2008, ApJ, 681, 375

Kratter, K. M., Matzner, C. D., Krumholz, M. R., \& Klein, R. I. 2010, ApJ, 708, 1585

Krumholz, M. R. 2006, ApJ, 641, L45

Krumholz, M. R., Klein, R. I., \& McKee, C. F. 2007, ApJ, 656, 959

-. 2012, ApJ, 754, 71

Krumholz, M. R., Klein, R. I., McKee, C. F., Offner, S. S. R., \& Cunningham, A. J. 2009, Science, 323, 754

Krumholz, M. R. \& McKee, C. F. 2008, Nature, 451, 1082

Krumholz, M. R., McKee, C. F., \& Klein, R. I. 2005, ApJ, 618, L33

Kuiper, R., Klahr, H., Beuther, H., \& Henning, T. 2010, ApJ, 722, 1556

-. 2011, ApJ, 732, 20 
Lamers, H. J. G. L. M. \& Cassinelli, J. P. 1999, Introduction to Stellar Winds, by Henny J. G. L. M. Lamers and Joseph P. Cassinelli, pp. 452. ISBN 0521593980. Cambridge, UK: Cambridge University Press, June 1999.,

Langer, N., Norman, C. A., de Koter, A., et al. 2007, A\&̋A, 475, L19

Lucy, L. B. \& White, R. L. 1980, ApJ, 241, 300

Lucy, L. B. \& Abbott, D. C. 1993, ApJ, 405, 738

Mauerhan, J. C., Smith, N., Filippenko, A., et al. 2012, arXiv:1209.6320

Massey, P., 2011, ASPC 440, 29

Massey, P., Zangari, A. M., Morrell, N. I., Puls, J., DeGioia-Eastwood, K., Bresolin, F., \& Kudritzki, R.-P. 2009, ApJ 692, 618

McKee, C. F. \& Tan, J. C. 2003, ApJ, 585, 850

de Mink, S. E., Langer, N., \& Izzard, R. G. 2011, Bulletin de la Societe Royale des Sciences de Liege, 80, 543

Moeckel, N. \& Clarke, C. J. 2011, MNRAS, 410, 2799

Mokiem, M. R., de Koter, A., Vink, J. S., Puls, J., Evans, C. J., Smartt, S. J., Crowther, P. A., Herrero, A., Langer, N., Lennon, D. J., Najarro, F., \& Villamariz, M. R. 2007, A\&广A 473, 603

Mokiem, M. R., de Koter, A., Evans, C. J., et al. 2007, A\&SA, 465, 1003

Muijres, L. E., Vink, J. S., de Koter, A., Müller, P. E., \& Langer, N. 2012, A\&3A, 537, A37

Müller, P. E. \& Vink, J. S. 2008, A\&A, 492, 493

Myers, A. T., Cunningham, A. J., Klein, R. I., Krumholz, M. R., \& McKee, C. F. 2012, ApJ, in preparation

Nakano, T., Hasegawa, T., \& Norman, C. 1995, ApJ, 450, 183

Nazé, Y. 2009, A\&A, 506, 1055

Nomoto, K. 2012, IAU Symposium, 279, 1

Nugis, T. \& Lamers, H. J. G. L. M. 2002, A\&SA, 389, 162

Ochsendorf, B. B., Ellerbroek, L. E., Chini, R., et al. 2011, A\& A, 536, L1

Oey, M. S. \& Clarke, C. J. 2005, ApJL, 620, L43

Oskinova, L. M. 2005, MNRAS, 361, 679

Oskinova, L. M., Hamann, W.-R., \& Feldmeier, A. 2007, A $₫ A, 476,1331$

Owocki, S. P. \& Gayley, K. G. 1997, in Astronomical Society of the Pacific Conference Series, Vol. 120, Luminous Blue Variables: Massive Stars in Transition, ed. A. Nota \& H. Lamers, 121

Owocki, S. P., Castor, J. I., \& Rybicki, G. B. 1988, ApJ, 335, 914

Owocki, S. P., Gayley, K. G., \& Shaviv, N. J. 2004, ApJ, 616, 525

Pauldrach, A. W. A., Vanbeveren, D., \& Hoffmann, T. L. 2012, A\& $A$, 538, A75

Petrovic, J., Pols, O., \& Langer, N. 2006, A\&SA, 450, 219

Pittard, J. M. \& Stevens, I. R. 2002, A\&A, 388, L20

Portegies Zwart, S. F., Makino, J., McMillan, S. L. W., \& Hut, P. 1999, A\& A, 348, 117

Puls, J., Urbaneja, M. A., Venero, R., et al. 2005, A\&A, 435, 669

Puls, J., Vink, J. S., \& Najarro, F. 2008, A\&ARv 16, 209

Quimby, R. M., Kulkarni, S. R., Kasliwal, M. M., et al. 2011, Nature, 474, 487

Rauw, G., De Becker, M., Nazé, Y., et al. 2004, A\&A, 420, L9

Rosen, A. L., Krumholz, M. R., \& Ramirez-Ruiz, E. 2012, ApJ, 748, 97

Rosolowsky, E. \& Simon, J. D. 2008, ApJ, 675, 1213

Sana, H., de Koter, A., de Mink, S. E., et al. 2012, arXiv:1209.4638

Sander, A., Hamann, W.-R., \& Todt, H. 2012, A\&A, 540, A144

Schaller, G., Schaerer, D., Meynet, G., \& Maeder, A. 1992, A\&ऽAS, 96, 269

Schnurr, O., Moffat, A. F. J., St-Louis, N., Morrell, N. I., \& Guerrero, M. A. 2008, MNRAS, 389,806

Schnurr, O., Moffat, A. F. J., Villar-Sbaffi, A., St-Louis, N., \& Morrell, N. I. 2009, MNRAS, 395,823

Shaviv, N. J. 2000, ApJ, 532, L137

Shaviv, N. J. 2001a, ApJ, 549, 1093

Shaviv, N. J. 2001b, MNRAS, 326, 126 
Smartt, S. J., Eldridge, J. J., Crockett, R. M., \& Maund, J. R. 2009, MNRAS, 395, 1409

Springmann, U. 1997, PhD thesis, Thesis, Ludwig-Maximilians-Universität München, (1997)

Sundqvist, J. O., Puls, J., \& Feldmeier, A. 2010, A\& $A$, 510, A11

Tramper, F., Sana, H., de Koter, A., \& Kaper, L. 2011, ApJ Letters 741, L8

Trundle, C., Dufton, P. L., Hunter, I., Evans, C. J., Lennon, D. J., Smartt, S. J., \& Ryans, R. S. I. $2007, A \& A 471,625$

Vanbeveren, D., De Donder, E., van Bever, J., van Rensbergen, W., \& De Loore, C. 1998, New Astronomy 3, 443

Vink, J. S. 2009, arXiv:0905.3338

Vink, J. S. \& Gräfener, G. 2012, ApJ, 751, L34

Vink, J. S., de Koter, A., \& Lamers, H. J. G. L. M. 2000, A\&\&A, 362, 295

Vink, J. S., de Koter, A., \& Lamers, H. J. G. L. M. 2001, $A \mathscr{E} A$, 369, 574

Vink, J. S., Muijres, L. E., Anthonisse, B., et al. 2011, A\& $A$, 531, A132

Voss, R., Diehl, R., Hartmann, D. H., et al. 2009, A\&GA, 504, 531

Voss, R., Diehl, R., Vink, J. S., \& Hartmann, D. H. 2010, A $\& A$, 520, A51

Voss, R., Martin, P., Diehl, R., et al. 2012, A\&SA, 539, A66

Weidner, C. \& Kroupa, P. 2004, MNRAS, 348, 187

Whitney, B. A., Wood, K., Bjorkman, J. E., \& Wolff, M. J. 2003, ApJ, 591, 1049

Wolfire, M. G. \& Cassinelli, J. P. 1987, ApJ, 319, 850

Woosley, S. E. \& Heger, A. 2012, ApJ, 752, 32

Woosley, S. E., Heger, A., \& Weaver, T. A. 2002, Reviews of Modern Physics, 74, 1015

Woosley, S. E., Blinnikov, S., \& Heger, A. 2007, Nature, 450, 390

Wright, A. E. \& Barlow, M. J. 1975, MNRAS, 170, 41

Yoon, S.-C., Dierks, A., \& Langer, N. 2012, A\&A, 542, A113

Yoon, S.-C., Langer, N., \& Norman, C. 2006, A\&A, 460, 199

Yoon, S.-C., Gräfener, G., Vink, J. S., Kozyreva, A., \& Izzard, R. G. 2012, A\& A, 544, L11

Yorke, H. W. \& Kruegel, E. 1977, A\&A, 54, 183

Yorke, H. W. \& Sonnhalter, C. 2002, ApJ, 569, 846

Yoshida, T. \& Umeda, H. 2011, MNRAS, 412, L78

Yungelson, L. R., van den Heuvel, E. P. J., Vink, J. S., Portegies Zwart, S. F., \& de Koter, A. $2008, A \& A, 477,223$

Zhang, Y. \& Tan, J. C. 2011, ApJ, 733, 55 